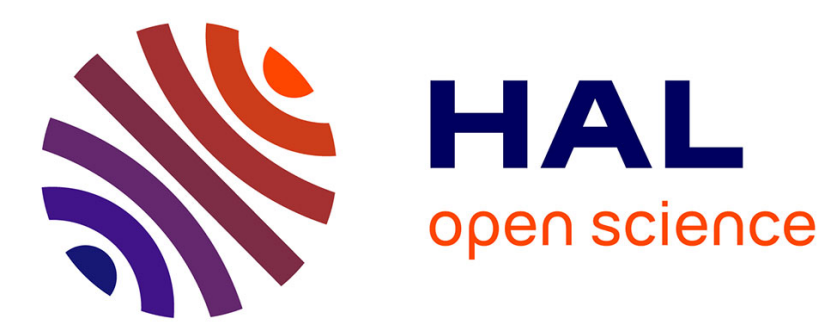

\title{
Topological gradient in structural optimization under stress and buckling constraints
}

\author{
Florian Mitjana, Sonia Cafieri, Florian Bugarin, Stéphane Segonds, F
}

Castanie, P Duysinx

\section{To cite this version:}

Florian Mitjana, Sonia Cafieri, Florian Bugarin, Stéphane Segonds, F Castanie, et al.. Topological gradient in structural optimization under stress and buckling constraints. Applied Mathematics and Computation, 2021, 409, 10.1016/j.amc.2021.126032 . hal-03134108

\section{HAL Id: hal-03134108 \\ https://hal.science/hal-03134108}

Submitted on 8 Feb 2021

HAL is a multi-disciplinary open access archive for the deposit and dissemination of scientific research documents, whether they are published or not. The documents may come from teaching and research institutions in France or abroad, or from public or private research centers.
L'archive ouverte pluridisciplinaire HAL, est destinée au dépôt et à la diffusion de documents scientifiques de niveau recherche, publiés ou non, émanant des établissements d'enseignement et de recherche français ou étrangers, des laboratoires publics ou privés. 


\title{
Topological gradient in structural optimization under stress and buckling constraints
}

\author{
F. Mitjana \\ HEC Montréal, GERAD, Canada \\ AVANTIS Project, Toulouse, France \\ S. Cafieri \\ ENAC, Université de Toulouse, F-31055 Toulouse, France \\ F. Bugarin, S. Segonds \\ Université de Toulouse, INSA/UPS/ISAE/Mines Albi, ICA UMR CNRS 5312, Toulouse, \\ 31400, France \\ F. Castanie \\ AVANTIS Project, Toulouse, France \\ P. Duysinx \\ University of Liège, Department of Aerospace and Mechanical Engineering, 4000, Liège, \\ Belgium
}

\begin{abstract}
Structural topology optimization aims to design mechanical structures by seeking the optimal material layout within a given design space. Within this framework, this paper addresses the minimization of the structural mass under stress and buckling constraints, formulated as a nonlinear combinatorial optimization problem. An algorithm is proposed for such a problem, that follows a topological gradient-based approach. The adjoint method is applied to efficiently compute the constraint gradients. An iterative algorithm for buckling analysis, featuring low memory requirements, is also proposed. Numerical results, including a real application arising in the aeronautical field, illustrate the efficiency of the two proposed algorithms.

Keywords: topology optimization, topological gradient, buckling constraints, generalized eigenvalue problem

Preprint submitted to Elsevier

January 24, 2021
\end{abstract}




\section{Introduction}

Topology optimization plays a prominent role in structural engineering, where one seeks the optimal distribution of a given material in a given design space. Applications arise typically in industry and include aircraft Re5 mouchamps et al. (2011); Zhu et al. (2015)) and automotive (Calvel (2004)) component design, and civil engineering (Kingman et al. (2015)).

In the context of continuum structures, a mathematical optimization problem and an associated method to compute the optimal topology are defined according to two main approaches. The first one consists in associating a design variable to each element of the mesh defining the design domain. Design variables may be continuous, representing the value of the density associated to each element, or binary, to signify the presence/absence of each element (where the values 1 and 0 can be interpreted as extreme values for densities). Associated methods are sometimes referred to as density-based methods (Bendsoe

15 \& Sigmund (2013); Deaton \& Grandhi (2014)). The second approach relies on implicit functions to define the structural boundaries. Associated methods are Level-set methods (Wang et al. (2003); Allaire et al. (2004)). In this paper, we consider an explicit parametrization of the design domain, that is discretized and meshed, and a binary variable is associated to each element. This yields to a combinatorial optimization problem. Topology optimization problems are characterized by constraints generally related to the inherent mechanical properties of the material and to the resistance of the structure under applied efforts in tension and compression. In this paper, we deal with stress and buckling constraints, and we aim at minimizing the structural mass.

Stress constraints are related to the integrity of the material under the applied external loads. Buckling constraints are related to the failure of a mechanical structure under instable phenomena due to compressive loads. From a mechanical point of view, these constraints play a crucial role in the behavior of the structure and have to be taken into account as soon as possible in the design 
process and so in the topological optimization (Duysinx et al. (2008); Shanglong et al. (2020) ). However, these kind of constraints, while of most importance in real applications, are difficult to handle in an optimization process, especially due to their non-linear nature.

Considering stress constraints, another difficulty is related to the fact that one of such constraints is associated to each of the elements of the discretized domain, so that there are as many design variables as there are constraints. Thus, the computational cost to handle such constraints may become critical for large-scale problems. To overcome this difficulty, aggregation techniques have been proposed, such as those based on the Kreisselmeier-Steinhauser function

40 (Kreisselmeier \& Steinhauser (1980)), the P-norm (Duysinx \& Sigmund (1998)) or a unified aggregation and relaxation approach (Verbart et al. (2017)). In this paper, we consider the formulation proposed by Verbart et al. (2017) and propose an efficient method to compute the derivatives of stress constraints.

Considering buckling phenomena in the context of topological optimization brings two kinds of difficulties: one related to the computational cost of the buckling analysis (Bian \& Fang (2017)) and the other one to the poor convergence of some optimization processes (Bruyneel et al. (2008); Gao et al. (2020)).

Buckling analysis of a structure is carried out through solving first a linear system to evaluate the structural displacements, and then a generalized eigenvalue problem implying two matrices. The first one is the stiffness matrix related to the structure, while the second one is the geometric stiffness matrix related to the deformations caused by the loading. The generalized eigenvalues represent the load factors of buckling, while the associated eigenvectors, related to displacements of the structure, are referred to as buckling modes. The smallest positive eigenvalue is called critical buckling load. For large scale problems, the buckling analysis can become highly memory and time consuming. Then, one challenge lies in an efficient method to solve the generalized eigenvalue problem. Krylov sub-space methods (Lehoucq et al. (1998)) and the Block Jacobi Conjugate Gradient method (Ovtchinnikov (2008)) lead to a very slow convergence due to the poor conditioning of the two involved matrices. Based on a shift-invert 
technique and a matrix factorization, Dunning et al. (2016) improved the rate of convergence of the Block Jacobi Conjugate Gradient method, but their aproach is highly memory consuming. The authors of Bian \& Fang (2017) proposed an assembly-free iterative solver to efficiently estimate the critical buckling load.

65 Their solver is based on the inverse iteration method, coupled with an efficient matrix-blocks computation that leads to low memory requirements.

In the context of structural optimization under buckling constraints, it is usual to compute only the critical buckling load as it is considered enough to assess the structural resistance to buckling (Lindgaard \& Dahl (2013); Luo 70 \& Tong (2015); Bian \& Fang (2017)). However, as highlighted by Bruyneel et al. (2008), as the topology of the structure changes during optimization, the buckling mode associated to the critical buckling load may also change, leading to a slow convergence or to divergence of the optimization process. Several „works (Bruyneel et al. (2008); Browne et al. (2012); Dunning et al. (2016); Gao

75 et al. (2020) ) showed that using a set of several eigenvalues in the optimization problem may overcome this difficulty. In this paper, building on the the inverse iteration method of Bian \& Fang (2017), we propose an extension of such a method that is able to efficiently compute multiple buckling modes.

Another difficulty of handling buckling constraints is related to the com80 putation of their derivatives. By construction, the geometric stiffness matrix depends on the decision variables and on the stress of elements. In order to reduce the computational cost, the partial derivatives with respect to the stress are often neglected (Neves et al. (1995); Bruyneel et al. (2008)). However, this approach affects the convergence of the optimization process. Although an explicit formulation of the derivatives of the geometric stiffness matrix has been proposed by Browne et al. (2012), their computation is highly computational expensive, thus untractable for large problems.

Most of topology optimization methods fall in the category of density-based methods. The most common one is the Solid Isotropic Material with Penal90 ization (SIMP), based on a continuous relaxation of binary decision variables. For problems under buckling constraints, the continuous relaxation implies ill- 
conditioning of the stiffness and the geometric stiffness matrices involved in the generalized eigenvalue problem, that may strongly impact the convergence of an iterative method (such as the inverse iteration method) for solving the generalized eigenvalue problem (Suresh (2013)). Moreover, the continuous relaxation may introduce undesirable buckling modes, called spurious buckling modes (Neves et al. (1995)).

A recent approach, which combines the SIMP method with the Non-Uniform Rational Basis Spline (NURBS) hyper-surfaces framework (Costa et al. (2018. $2019 \mathrm{~b})$ ), allows one to describe the topology of a continuum structure through a geometrical entity. The number of design variables is reduced with respect to density-based methods, yielding an interesting advantage to address large-scale problems. This approach has recently been proved to efficiently handle constraints such as minimum and maximum length scale (Costa et al. (2019a c)), structural displacements (Rodriguez et al. (2020)) or eigenfrequencies (Costa \& Montemurro (2020)); it has also been applied to multi-material topology optimization problems (Gao et al. $(2020 \mathrm{a} b))$. Thanks to the geometrical properties of NURBS hyper-surfaces, it is possible to implicitly include in the problem formulation mechanical requirements such as buckling constraints. However, as this method is based on the SIMP approach, buckling analysis is still impacted by ill-conditioning of the stiffness and the geometric stiffness matrices.

Another optimization approach, that is receiving an increasing attention (Suresh \& Takalloozadeh (2013); Deng \& Suresh (2015)), relies on topological gradients. Unlike the SIMP method, this kind of approach is not based on a problem relaxation, thus overcoming the problem of ill-conditioning of matrices in the generalized eigenvalue problem.

In this paper, a new algorithm, based on topological gradients, for solving topology optimization problems under stress and buckling constraints is presented. Our contributions are also related to the way stress and buckling constraints are handled. As regards to stress constraints, the formulation proposed by Verbart et al. (2017) is considered and a method to compute constraint derivatives by introducing an adjoint state is proposed. Concerning buckling 
analysis, the method based on the iterative inverse (Bian et al. (2015)) is extended to compute a broader spectrum of eigenvalues. From the formulation of the buckling constraint of Browne et al. (2012), we propose a method based on adjoint states to compute efficiently the associated buckling derivatives.

The article is organized as follows. In Section 2, the optimization problem formulation is presented. In Section 3, an extension of the iterative inverse method is proposed to perform the buckling analysis. Section 4 presents the optimization method proposed for the considered problem. Numerical results are presented in Section 5. Finally, Section 6 concludes the article.

\section{Optimization problem modeling}

In this section, we present the formulation of the topology optimization problem for continuous structures addressed in this paper. The design space, 135 i.e., the design domain of the structure, is discretized in a regular grid of $N$ cube elements in a three-dimensional space. Vertices of the cube elements are called nodes, and the total number of nodes is denoted by $M$. A binary variable $x_{i}$ is associated to each element $i$ of the mesh meaning its presence or absence. These decision variables will also be referred to as design variables.

We aim at optimizing the structural design in such a way that the mass of the structure is minimized. The structural mass is defined by the material density $\rho$ multiplied by the sum of the design variables, each multiplied by the volume $\omega$ of the corresponding element. As in our model all the elements have equal volume, we can define the objective function, to be minimized, as follows:

$$
\rho \omega \sum_{i=1}^{N} x_{i}
$$

As for the constraints, let us formulate the stress and the buckling constraints that characterize the problem under investigation. The evaluation of the structural stress and buckling first requires the solution of the following linear system:

$$
\mathbf{K u}=\mathbf{f}
$$


where $\mathbf{K} \in \mathbb{R}^{3 M \times 3 M}$ is the stiffness matrix, $\mathbf{u} \in \mathbb{R}^{3 M}$ is the displacement vector of the $M$ nodes (each node has 3 possible displacements along the axes of the space, noted as $\mathrm{x}, \mathrm{y}$ and $\mathrm{z}), \mathbf{f} \in \mathbb{R}^{3 M}$ represents the loads applied on the structure. Note that one actually solves a system whose size is lower than $3 M \times 3 M$. It is indeed easy to see that, for example, in order to keep fixed a portion of the structure, the associated nodal displacements have to be given a value of 0 . More generally, boundary conditions on some nodal displacements must be imposed, thus reducing the size of (2). Once the vector $\mathbf{u}$ has been computed, the stress components $\left\{\sigma_{\mathrm{x}}^{i}, \sigma_{\mathrm{y}}^{i}, \sigma_{\mathrm{z}}^{i}, \sigma_{\mathrm{xy}}^{i}, \sigma_{\mathrm{xz}}^{i}, \sigma_{\mathrm{yz}}^{i}\right\}$ of a structural element $i$ can be evaluated in its midpoint as follows (the explicit dependence of each component from $\mathbf{x}$ and $\mathbf{u}$ being omitted for the sake of readability):

$$
\left[\sigma_{\mathrm{x}}^{i}, \sigma_{\mathrm{y}}^{i}, \sigma_{\mathrm{z}}^{i}, \sigma_{\mathrm{xy}}^{i}, \sigma_{\mathrm{xz}}^{i}, \sigma_{\mathrm{yz}}^{i}\right]^{\top}=\mathbf{H B}_{i} \mathbf{u}_{i}
$$

where $\mathbf{u}_{i}$ corresponds to the displacements of the nodes of the element $i, \mathbf{H}$ is the Hooke's matrix computed from the problem data (material property) and $\mathbf{B}_{i}$ is a matrix related to the geometry of the element $i$ resulting from the discretization (Cook et al. (1974)).

From equation (3), the Von Mises stress $\sigma_{i}^{V M}$ of an element $i$ can then be expressed, as a function of $\mathbf{x}=\left\{x_{1}, \ldots, x_{N}\right\}$ and $\mathbf{u}$, as follows:

$$
\sigma_{i}^{V M}(\mathbf{x}, \mathbf{u})=\frac{1}{\sqrt{2}} \sqrt{\left(\sigma_{\mathrm{x}}^{i}-\sigma_{\mathrm{y}}^{i}\right)^{2}+\left(\sigma_{\mathrm{x}}^{i}-\sigma_{\mathrm{z}}^{i}\right)^{2}+\left(\sigma_{\mathrm{y}}^{i}-\sigma_{\mathrm{z}}^{i}\right)^{2}+6\left(\left(\sigma_{\mathrm{xy}}^{i}\right)^{2}+\left(\sigma_{\mathrm{xz}}^{i}\right)^{2}+\left(\sigma_{\mathrm{yz}}^{i}\right)^{2}\right)}
$$

Following Verbart et al. (2017), we finally formulate the stress constraint as:

$$
G_{\sigma}(\mathbf{x}, \mathbf{u}):=\frac{1}{P} \ln \left(\frac{1}{N} \sum_{i=1}^{N} e^{P g_{i}(\mathbf{x}, u)}\right) \leq 0, \text { where } g_{i}(\mathbf{x}, \mathbf{u})=x_{i}\left(\frac{\sigma_{i}^{V M}(\mathbf{x}, \mathbf{u})}{\bar{\sigma}}-1\right)
$$

where $\bar{\sigma}$ is a constant inherent to the considered material, referred to as limit stress, and $P$ represents an aggregation parameter (Verbart et al. (2017)). When $P$ goes to infinity, this function converges to the maximal value of $g_{i}$.

From the stress components in (3), the geometric stiffness matrix $\mathbf{K}_{\sigma} \in \mathbb{R}^{n \times n}$ can be assembled taking into account the blocks corresponding to each element 
$i$, through the following expression:

$$
\mathbf{K}_{\sigma}=\sum_{i=1}^{N}\left[\int_{\boldsymbol{\Omega}_{i}} \mathbf{G}^{\top}\left(\begin{array}{ccc}
\boldsymbol{\sigma}_{i} & 0 & 0 \\
0 & \boldsymbol{\sigma}_{i} & 0 \\
0 & 0 & \boldsymbol{\sigma}_{i}
\end{array}\right) \mathbf{G} d \mathrm{x} d \mathrm{y} d \mathbf{z}\right] \text { where } \boldsymbol{\sigma}_{i}(\mathbf{x}, \mathbf{u})=\left(\begin{array}{ccc}
\sigma_{\mathrm{x}}^{i} & \sigma_{\mathrm{xy}}^{i} & \sigma_{\mathrm{xz}}^{i} \\
\sigma_{\mathrm{xy}}^{i} & \sigma_{\mathrm{y}}^{i} & \sigma_{\mathrm{yz}}^{i} \\
\sigma_{\mathrm{xy}}^{i} & \sigma_{\mathrm{yz}}^{i} & \sigma_{\mathrm{z}}^{i}
\end{array}\right)
$$

where $\boldsymbol{\Omega}_{i}$ represents the volume of element $i$ and $\mathbf{G}$ is a matrix whose components can be computed from the discretization of the meshed domain (Cook et al. (1974)), and [ ] represents the block assembly process. Thus, we are able to define the generalized eigenvalues problem related to buckling:

$$
\left(\mathbf{K}+\lambda \mathbf{K}_{\sigma}\right) \mathbf{v}=0
$$

where $\lambda$ are scaling factors (critical loads) of the applied forces $\mathbf{f}$, and the associated eigenvectors $\mathbf{v}$ represent the corresponding deformations of the structure (buckling modes).

To ensure that the structure resists to buckling, one needs to ensure that the critical buckling load $\lambda_{c r}$ is larger than a safety coefficient $c_{s}$. Although using the critical buckling load $\lambda_{c r}$ is enough to assess the structural stability, several works (Bruyneel et al. (2008); Browne et al. (2012); Dunning et al. (2016); Gao et al. (2020) showed that using a set of several buckling modes allows one to avoid oscillations and slow convergence of the optimization process. In Browne et al. (2012), the authors use the positive semi-definite property of the stiffness matrix to formulate a unique buckling constraint which takes into account several buckling modes. Following Browne et al. (2012), we formulate the buckling constraint as follows:

$$
G_{\lambda}:=\frac{1}{m} \sum_{j=1}^{m} \mathbf{v}_{j}^{\top}\left(\mathbf{K}+c_{s} \mathbf{K}_{\sigma}\right) \mathbf{v}_{j} \geq 0
$$

where the vectors $\mathbf{v}_{j}$ are the $m$ buckling modes of the generalized eigenvalue problem (7) and $c_{s}$ is the safety coefficient.

The problem of mass minimization under stress and buckling constraints can finally be summarized as the following nonlinear combinatorial optimization problem: 


$$
\begin{array}{ll}
\min _{\mathbf{x}=\left(x_{1}, \ldots, x_{N}\right)} & \rho \omega \sum_{i=1}^{N} x_{i} \\
\text { s.t. } & \frac{1}{P} \ln \left(\frac{1}{N} \sum_{i=1}^{N} e^{P . g_{i}(\mathbf{x}, \mathbf{u})}\right) \leq 0, \quad \text { where } g_{i}(\mathbf{x}, \mathbf{u})=x_{i}\left(\frac{\sigma_{i}^{V M}(\mathbf{x}, \mathbf{u})}{\bar{\sigma}}-1\right) \\
& \frac{1}{m} \sum_{j=1}^{m} \mathbf{v}_{j}^{\top}\left(\mathbf{K}+c_{s} \mathbf{K}_{\sigma}\right) \mathbf{v}_{j} \geq 0 \\
& \mathbf{K u}=\mathbf{f} \\
& \left(\mathbf{K}+\lambda \mathbf{K}_{\sigma}\right) \mathbf{v}=0 \\
& x_{i} \in\{0,1\} \forall i=1, \ldots, N .
\end{array}
$$

\section{Linear buckling analysis}

In this section, we propose an extension of the inverse iteration method developed by Bian \& Fang (2017) to compute the $m$ buckling eigenvectors associated to equation (8) (defining buckling constraints of the optimization problem $(\mathrm{P})$ ). Let us first briefly recall the main elements of the method. For further details, please refer to Bian \& Fang (2017). The method aims at avoiding assembly of the stiffness and of the geometric stiffness matrices, $\mathbf{K}$ and $\mathbf{K}_{\sigma}$, in order to reduce memory cost. The main operation, relying on a matrix-vector multiplication, is performed as a matrix-blocks computation (Hughes et al. (1983)). As an example, considering the stiffness matrix $\mathbf{K}$ and the structural displacement vector $\mathbf{u}$, the matrix-vector multiplication $\mathbf{K u}$ is performed as:

$$
\mathbf{K u}=\sum_{i=1}^{N}\left[\mathbf{K}_{i} \mathbf{u}_{i}\right]
$$

where $\mathbf{K}_{i}$ is the elementary stiffness matrix of element $i, \mathbf{u}_{i}$ are the displacements of the nodes of element $i$ and [ ] represents the block assembly process of the $\mathbf{K}_{i} \mathbf{u}_{i}$ vectors in the $\mathbf{K u}$ vector. In the case of a regular mesh grid, meaning that all finite elements are identical (as it is in our case), the matrices $\mathbf{K}_{i}$ are 
identical for all $i$. Thus, for a stiffness matrix-vector multiplication, only the stiffness matrix $\mathbf{K}_{i}$ of an element needs to be stored, leading to low memory requirements (Yadav \& Suresh (2014)).

The computational cost is also reduced through the application of the deflated conjugate gradient method (Saad et al. (2000) ) that appears to be particularly efficient to solve the (sparse) linear systems in the considered context. More precisely, Bian \& Fang (2017) used the deflated conjugate gradient method within an inverse iteration method (Ipsen (1997); Golub \& Ye (2000) $)$, that they adapted to the generalized eigenvalue problem (7) to determine the critical buckling load $\lambda_{c r}$ and its associated eigenvector $\mathbf{v}_{c r}$. The method consists in solving iteratively the following linear system starting from an initial approximation $v_{0}$ :

$$
\mathbf{v}_{k+1}=-\mathbf{K}^{-1} \mathbf{K}_{\sigma} \mathbf{v}_{k}
$$

where the vector $\mathbf{v}_{k}$ is normalized at each iteration. Once the sequence $\mathbf{v}_{k}$ has converged to an eigenvector $\mathbf{v}$, we can compute the associated eigenvalue $\lambda$ by the Rayleigh quotient functional (Timoshenko \& Gere (1960)) related to the generalized eigenvalue problem (7):

$$
R(v)=-\frac{\mathbf{v}^{\top} \mathbf{K} \mathbf{v}}{\mathbf{v}^{\top} \mathbf{K}_{\sigma} \mathbf{v}}
$$

It is important to note that the eigenvalue $\lambda$ corresponds to the eigenvalue with the smallest modulus of the generalized eigenvalue problem (7). If we assume that the geometric stiffness matrix is positive definite, then the eigenvalue $\lambda$ always corresponds to the critical buckling load $\lambda_{c r}$. However, as it is not necessarily the case, the eigenvalue $\lambda$ might correspond either to the smallest positive eigenvalue or to the smallest negative eigenvalue. Thus, in the context of structural optimization, this method can be applied only when the smallest eigenvalue has a positive modulus.

We propose to extend the inverse iteration method to compute a set of $m$ positive eigenvalues of the generalized eigenvalue problem (7). The method is based on the minimization of the Rayleigh quotient functional of a set of vectors. First note that the minimization of the Rayleigh quotient of $m$ vectors 
will converge to the $m$ associated eigenvalues of smallest moduli. However, as noted above, the geometric stiffness matrix is not necessarily positive definite, so the associated eigenvalues are not always positive. To overcome this problem, let us consider the set $\Lambda$ of the $\widetilde{m}>m$ eigenvalues of smallest moduli:

$$
\Lambda:=\left\{\lambda_{j}, \forall j=1, \ldots, \tilde{m}|| \lambda_{1}|\leq| \lambda_{2}|\leq \cdots \leq| \lambda_{\tilde{m}-1}|\leq| \lambda_{\tilde{m}} \mid\right\}
$$

Assuming that $m$ positive eigenvalues exist for the considered problem (as is the case with most real-world applications in the field of structural optimization), and assuming that the cardinality of set $\Lambda$ is large enough, we can extract the subset of $m$ smallest positive eigenvalues. Thus, in order to determine the $m$ eigenvectors associated with the $m$ smallest positive eigenvalues, we propose to apply the inverse iteration method on a set $\mathbf{V}$ of $\tilde{m}$ vectors of size $3 M$ :

$$
\mathbf{V} \in \mathbb{R}^{3 M \times \widetilde{m}}:=\left\{\mathbf{v}_{1}, \ldots, \mathbf{v}_{\widetilde{m}}\right\}
$$

When solving the equation 10 for each vector of $\mathbf{V}$, it is necessary to maintain the $\mathbf{K}$-orthogonality of the vectors (Dunning et al. (2016)), otherwise each component of $\mathbf{V}$ will converge to the eigenvector associated to the eigenvalue of smallest modulus. In order to maintain the $\mathbf{K}$-orthogonality between the vectors belonging to $\mathbf{V}$, we propose to use the Rayleigh-Ritz procedure (Trefethen \& Bau III (1997) ), consisting in solving the generalized eigenvalue problem, of dimension $\tilde{m}$, as follows:

$$
\mathbf{V}^{\top} \mathbf{K}_{\sigma} \mathbf{V w}=\bar{\lambda} \mathbf{V}^{\top} \mathbf{K} \mathbf{V} \mathbf{w}
$$

where the vectors $\overline{\mathbf{v}}=\mathbf{V} \mathbf{w}$ are called Ritz vectors (where $\mathbf{w}$ are the eigenvectors of 12 ) and the eigenvalues $\bar{\lambda}$ are called Ritz values. Note that the Ritz vectors are $\mathbf{K}$-orthogonal by construction. Moreover the system $\sqrt{12}$ is much simpler to solve than the initial problem (7) since $\tilde{m}<<3 M$. This kind of problems can be solved by standard numerical linear algebra solvers as those implemented in the LAPACK library (Anderson et al. (1999)).

The procedure that we propose to compute the set of $m$ eigenvalues and eigenvectors of system (7) is summarized in Algorithm 1. Note that the Algorithm 1 stops when the $m$ smallest positive eigenvalues have been determined. 


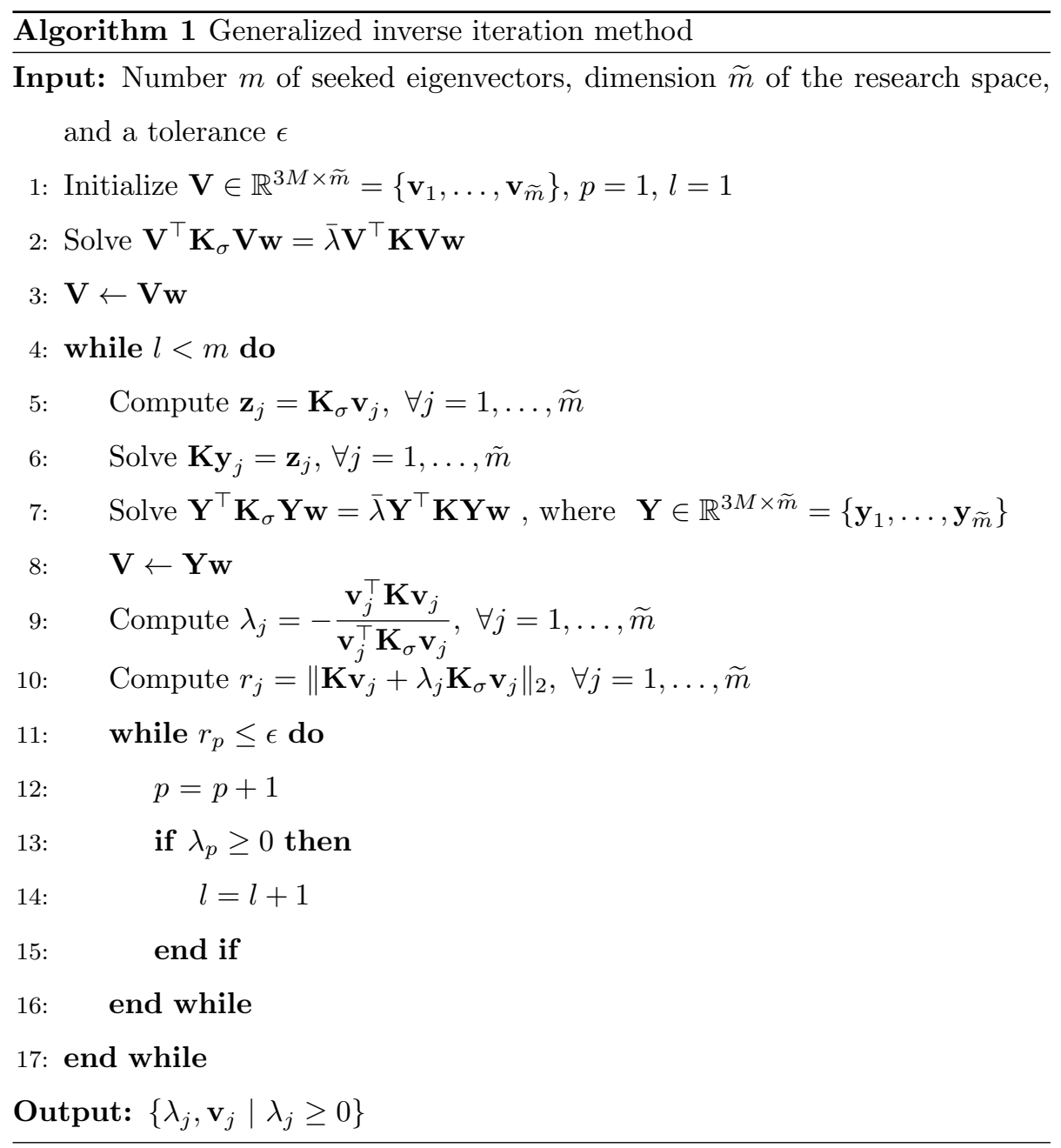

\section{Topological gradient-based optimization}

In this section, we propose an algorithm for topology optimization of a prob-

180 gradient. After a brief recall of such a concept and of its use within an optimization algorithm, we focus the stress and buckling constraints of the optimization problem formulated in Section 2. In an unified framework, we present a computation of topological gradients for the stress constraints, and of gradients for 


\subsection{Topological gradient framework}

Topology optimization problems rely on identifying optimal material distributions. Algorithms for topology optimization of mechanical structures are, thus, basically based on a principle of iterative material removal, starting from an initial domain.

The notion of topological gradient, introduced by Eschenauer et al. (1994), quantifies the influence, on an optimization criterion, of a perturbation of the design domain. From the point of view of a mechanical structure, this can be seen as the insertion of a hole in the structure, corresponding to an elementary amount of material that would be removed from the initial domain.

Let us consider a spatial domain $\boldsymbol{\Omega}$ and let $\boldsymbol{\Omega}_{B} \subset \boldsymbol{\Omega}$ be the domain obtained perturbing $\boldsymbol{\Omega}$ by the introduction of a ball $B\left(p_{i}, r\right)$ of radius $r$ centered in a point $p_{i} \in \boldsymbol{\Omega}$. Given an optimization criterion $C: \Omega \mapsto \mathbb{R}$, the topological gradient of $C$ in $p_{i}$ is defined as (Sokolowski \& Zochowski (1999); Céa et al. (2000); Novotny et al. (2007)):

$$
T_{C}\left(p_{i}\right)=\lim _{r \rightarrow 0} \frac{C\left(\boldsymbol{\Omega}_{B}\right)-C(\boldsymbol{\Omega})}{h(r)}
$$

where $h(r)$ is a positive scalar function such that $\lim _{r \rightarrow 0} h(r)=0$.

Topological gradients can then be used within topology optimization algorithms to define the principle of iterative material removal. Starting from a design domain that is initially considered full of material, and associated to a volume $\alpha$ equal to 1 , a straightforward approach consists in fixing a threshold $\tau$ and removing the fraction of volume $\Delta \alpha$ corresponding to the elements for which the topological gradient of the optimization criterion is less than $\tau$. Thus, the quantity of removed material can be a different one at each iteration, as it depends on the (fixed) value of $\tau$. Despite its simplicity, this kind of approach is not generally used in structural optimization, as it leads to a poor convergence of the optimization process (Suresh (2010)).

Another approach relies on fixing the fraction of volume $\Delta \alpha$ and determining the threshold $\tau$ such that the amount of volume corresponding to the elements whose topological gradient is less than $\tau$ is equal to $\Delta \alpha$ (Deng \& Suresh (2015)). 
Thus, unlike the previous approach, the value of $\tau$ is adapted throughout the optimization process. More specifically, given a domain $\Omega$ whose volume is $\alpha$, and given a fraction $\Delta \alpha$ of volume to remove, one has to determine a scalar $\tau$, that can be assimilated to a cut-off value, in order to obtain a perturbed domain $\Omega^{\tau}$ whose volume is $\alpha-\Delta \alpha$. The value of $\tau$ can be determined from a topo215 logical gradient field $T$ by identifying the elements whose associated topological gradient is larger than $\tau$, while ensuring that the volume is equal to $\alpha-\Delta \alpha$. Within an optimization algorithm, the value of the fraction of volume to remove is usually iteratively decreased, and iterations are run until a termination criterion is satisfied. The feasibility of the current topology for the addressed structure, i.e., the satisfaction of the constraints of the optimization problem, is checked at each iteration. This guarantees that a feasible, local albeit not global optimal solution, is always computed. Note that the value of the fraction of volume $\Delta \alpha$ plays a crucial role, since it leads the entire optimization process. Indeed, if its initial value is very small (for example, less than $1 \%$ of the initial 225 volume) then the convergence will be slow, while if its value is large (for example, more than $30 \%$ of the volume) then the optimization may diverge (Suresh (2013)).

An algorithmic scheme for structural optimization based on topological gradients is displayed in Figure 1. 


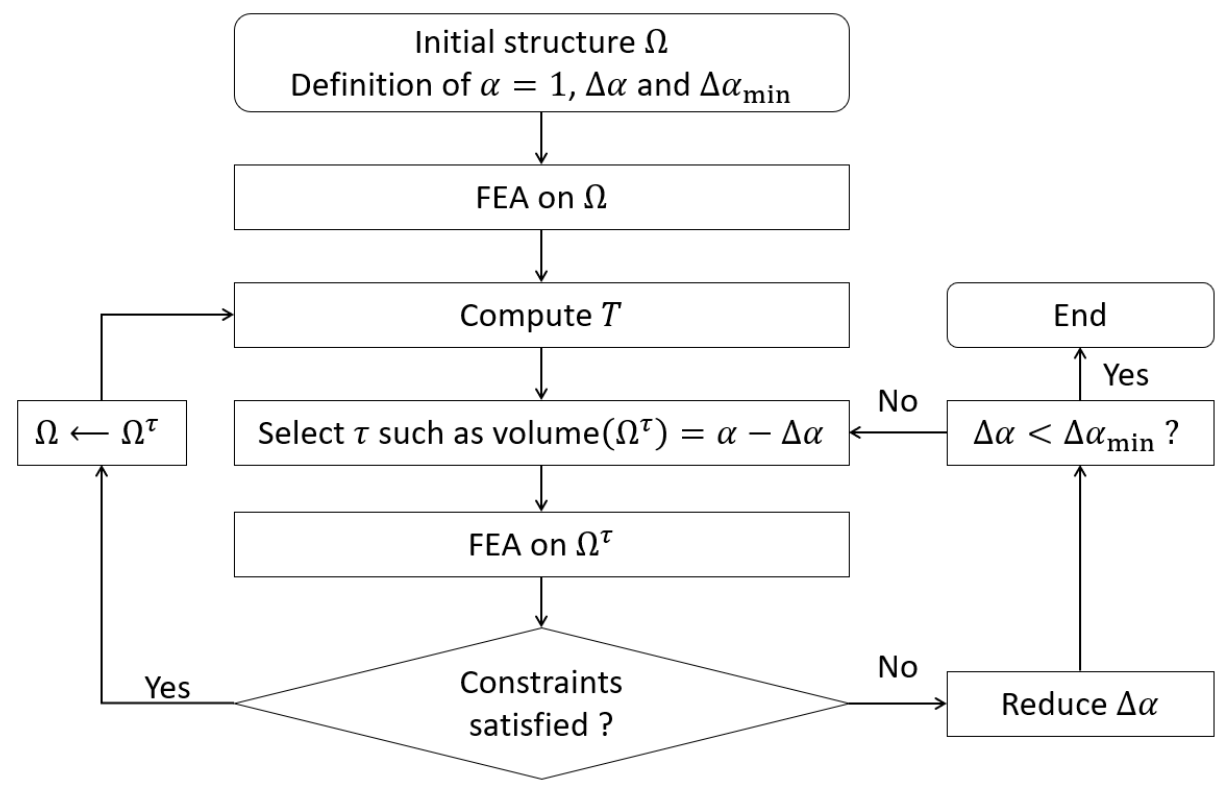

Figure 1: Optimization algorithm based on topological gradients

\subsection{Computing gradients for stress and buckling constraints}

Our aim is to devise a topological optimization algorithm for our problem $(P)$, in the algorithmic framework presented in the above subsection 4.1. Thus, in the following we focus on the stress and the buckling constraints $G_{\sigma}$ and $G_{\lambda}$, and we present a computation of gradients of such constraints. For such a computation, our decision variables $x_{i}$ are relaxed as $x_{i} \in[0,1], \forall i$, similarly to what is done in the framework of BESO methods (Huang \& Xie $(2009,2010)$; Deaton \& Grandhi (2014)).

We compute a topological gradient, $T_{\sigma}$, associated to the constraint $G_{\sigma}$, and a gradient, $T_{\lambda}$, associated to $G_{\lambda}$. They both will be used to estimate the in-

240 fluence of a perturbation introduced on the structure whose topology is being optimized. The computation of $T_{\sigma}$ and $T_{\lambda}$ represents one of the contributions of this paper. Topological gradients have indeed been introduced in the literature for compliance constraints (Feijoo et al. (2005)) and for the P-norm of stress constraints (Suresh \& Takalloozadeh $(2013))$, while constraints like $G_{\sigma}$ and $G_{\lambda}$ 245 have not been previously addressed in this context. Our approach to determine 
${ }_{\square} T_{\sigma}$ and $T_{\lambda}$ is based on the adjoint state method (Choi \& Kim (2006); Deng \& Suresh (2017); Allaire (2015)), as described below.

\subsubsection{Stress constraints}

Let us consider the stress constraint $G_{\sigma}$. As it depends on the decision variables and on the structural displacements $u$, its derivative according to the decision variable $x_{i}$ is expressed as:

$$
\frac{d G_{\sigma}}{d x_{i}}=\frac{\partial G_{\sigma}}{\partial x_{i}}+\frac{\partial G_{\sigma}}{\partial \mathbf{u}} \frac{d \mathbf{u}}{d x_{i}}
$$

The structural displacements are obtained throught the equation $K \mathbf{u}=f$ (see (2)). Thus by deriving according to $x_{i}$, we obtain:

$$
\mathbf{K} \frac{d \mathbf{u}}{d x_{i}}+\frac{\partial \mathbf{K}}{\partial x_{i}} u=0 \Leftrightarrow \frac{d \mathbf{u}}{d x_{i}}=-\mathbf{K}^{-1} \frac{\partial \mathbf{K}}{\partial x_{i}} \mathbf{u}
$$

Then, the derivative of $G_{\sigma}$ according to $x_{i}$ can be formulated as:

$$
\frac{d G_{\sigma}}{d x_{i}}=\frac{\partial G_{\sigma}}{\partial x_{i}}-\frac{\partial G_{\sigma}}{\partial \mathbf{u}} \mathbf{K}^{-1} \frac{\partial \mathbf{K}}{\partial x_{i}} \mathbf{u}
$$

It is important to note that, to solve equation (16), we need to solve a linear system involving the stiffness matrix $\mathbf{K}$. Then, computing the derivatives of $G_{\sigma}$ according to the decision variables $x_{i}, \forall i=1, \ldots, N$, requires the solution of $N$ linear systems, implying a potentially high computational cost. An alternative is represented by the adjoint method, that relies on the fact that the term $-\frac{\partial G_{\sigma}}{\partial \mathbf{u}} \mathbf{K}^{-1}$ does not depend on the decision variables and remains constant regardless of the variable $x_{i}$ in 16 . The adjoint method consists in defining an adjoint variable $\boldsymbol{\mu}$ (Choi \& Kim (2006)), solution of the following adjoint state:

$$
\mathbf{K} \boldsymbol{\mu}=-\frac{\partial G_{\sigma}}{\partial \mathbf{u}}
$$

where $\mathbf{K}$ is the stiffness matrix, $\boldsymbol{\mu} \in \mathbb{R}^{3 M}$ is the adjoint state and $\mathbf{u}$ corresponds to a vector of displacements. Then, the computation of the derivatives of $G_{\sigma}$ according to $x_{i}, \forall i=1, \ldots, N$ involves only one additional linear system. 
From the formulation of $G_{\sigma}$ in the equation (5), the term $-\frac{\partial G_{\sigma}}{\partial \mathbf{u}}$ can be expressed as:

$$
-\frac{\partial G_{\sigma}}{\partial \mathbf{u}}=-\frac{1}{P} \frac{1}{\frac{1}{N} \sum_{i=1}^{N} e^{P . g_{i}(\mathbf{x}, \mathbf{u})}} \frac{1}{N} \sum_{i=1}^{N}\left[P \frac{x_{i}}{\bar{\sigma}} e^{P \cdot g_{i}(\mathbf{x}, \mathbf{u})} \frac{\partial \sigma_{i}^{V M}}{\partial \mathbf{u}}\right]
$$

where [ ] corresponds to the block assembly operator. From (4), the derivative of $\sigma_{i}^{V M}$ with respect to a displacement $u$ can be expressed as:

$$
\begin{aligned}
\frac{\partial \sigma_{i}^{V M}}{\partial \mathbf{u}}= & \frac{1}{\sigma_{i}^{V M} \sqrt{2}}\left(\left(\sigma_{\mathrm{x}}^{i}-\sigma_{\mathrm{y}}^{i}\right)\left(\frac{\partial \sigma_{\mathrm{x}}^{i}}{\partial \mathbf{u}}-\frac{\partial \sigma_{\mathrm{y}}^{i}}{\partial \mathbf{u}}\right)+\left(\sigma_{\mathrm{x}}^{i}-\sigma_{\mathrm{z}}^{i}\right)\left(\frac{\partial \sigma_{\mathrm{x}}^{i}}{\partial \mathbf{u}}-\frac{\partial \sigma_{\mathrm{z}}^{i}}{\partial \mathbf{u}}\right)\right. \\
& \left.+\left(\sigma_{\mathrm{y}}^{i}-\sigma_{\mathrm{z}}^{i}\right)\left(\frac{\partial \sigma_{\mathrm{y}}^{i}}{\partial \mathbf{u}}-\frac{\partial \sigma_{\mathrm{z}}^{i}}{\partial \mathbf{u}}\right)+6\left(\sigma_{\mathrm{xy}}^{i} \frac{\partial \sigma_{\mathrm{xy}}^{i}}{\partial \mathbf{u}}+\sigma_{\mathrm{xz}}^{i} \frac{\partial \sigma_{\mathrm{xz}}^{i}}{\partial \mathbf{u}}+\sigma_{\mathrm{yz}}^{i} \frac{\partial \sigma_{\mathrm{yz}}^{i}}{\partial \mathbf{u}}\right)\right)
\end{aligned}
$$

Note that the derivatives of the stress components $\sigma_{\mathrm{x}}^{i}, \sigma_{\mathrm{y}}^{i}, \sigma_{\mathrm{z}}^{i}, \sigma_{\mathrm{xy}}^{i}, \sigma_{\mathrm{xz}}^{i}$ and $\sigma_{\mathrm{yz}}^{i}$ with respect to a displacement $\mathbf{u}$ are constant and correspond to :

$$
\begin{array}{llll}
\frac{\partial \sigma_{\mathrm{x}}^{i}}{\partial \mathbf{u}}=\mathbf{F}_{1,:} & \frac{\partial \sigma_{\mathrm{y}}^{i}}{\partial u}=\mathbf{F}_{2,:} & \frac{\partial \sigma_{\mathrm{z}}^{i}}{\partial \mathbf{u}}=\mathbf{F}_{3,:} \\
\frac{\partial \sigma_{\mathrm{xy}}^{i}}{\partial \mathbf{u}}=\mathbf{F}_{4,:} & \frac{\partial \sigma_{\mathrm{xz}}^{i}}{\partial \mathbf{u}}=\mathbf{F}_{5,:} & \frac{\partial \sigma_{\mathrm{yz}}^{i}}{\partial \mathbf{u}}=\mathbf{F}_{6,:}
\end{array}
$$

where $\mathbf{F}=\mathbf{H B} \in \mathbb{R}^{6 \times 24}$, with $\mathbf{H}$ and $\mathbf{B}$ matrices introduced in (3). In the case of a cube element (as those considered in our domain discretization), $\mathbf{B}_{i} \in \mathbb{R}^{6 \times 24}$ and the terms $\mathbf{F}_{i,:} \forall i=1, \ldots, 6$ correspond to to the rows of the $\mathbf{F}$ matrix. Thus, the term $-\frac{\partial G_{\sigma}}{\partial \mathbf{u}}$ can be expressed as:

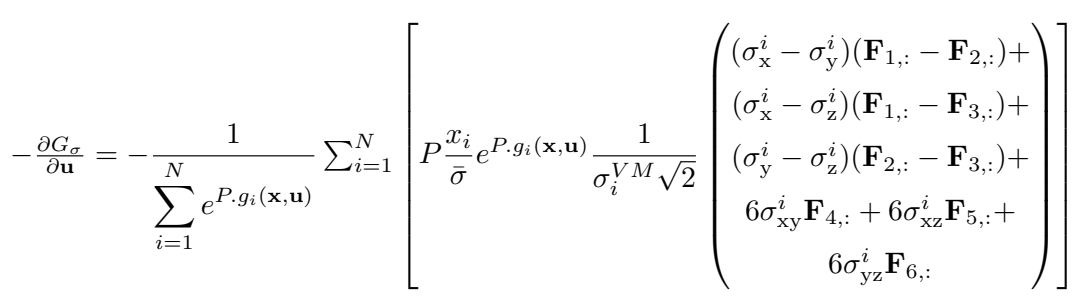

Then, the adjoint state $\boldsymbol{\mu}$ can be determined by solving the adjoint system (17) by the deflated conjugate gradient method. Thus, the topological gradient $T_{\sigma}$ of an element $x_{i}$ is expressed as (Feijoo et al. (2005)):

$$
T_{\sigma}\left(x_{i}\right)=-\frac{4}{1+\nu} \boldsymbol{\sigma}\left(\mathbf{u}\left(x_{i}\right)\right): \boldsymbol{\epsilon}\left(\boldsymbol{\mu}\left(x_{i}\right)\right)+\frac{1-3 \nu}{1-\nu^{2}} \operatorname{tr}\left(\boldsymbol{\sigma}\left(\mathbf{u}\left(x_{i}\right)\right)\right) \operatorname{tr}\left(\boldsymbol{\epsilon}\left(\boldsymbol{\mu}\left(x_{i}\right)\right)\right)
$$


where $\nu$ is the Poisson ratio, $\boldsymbol{\sigma}\left(\mathbf{u}\left(x_{i}\right)\right)$ is the stress matrix, as noted in (6), according to the vector of displacements $u$ of the element $i$ and $\boldsymbol{\epsilon}\left(\boldsymbol{\mu}\left(x_{i}\right)\right)$ is the deformation matrix of the $\boldsymbol{\mu}$ adjoint vector of the $i$ element. The operator : corresponds to the sum of the matrix components obtained from the Hadamard product. The $t r$ operator is the trace operator of a matrix.

\subsubsection{Buckling constraints}

Let us now consider the buckling constraint $G_{\lambda}$. First, observe that the derivative of $G_{\lambda}$ with respect to $x_{i}$ can be expressed as:

$$
\frac{d G_{\lambda}}{d x_{i}}=\frac{1}{m} \sum_{i=1}^{m} 2 \mathbf{v}_{i}^{\top}\left(\mathbf{K}+c_{s} \mathbf{K}_{\sigma}\right) \frac{d \mathbf{v}_{i}}{d x_{i}}+\mathbf{v}_{i}^{\top}\left(\frac{d \mathbf{K}}{d x_{i}}+c_{s} \frac{d \mathbf{K}_{\sigma}}{d x_{i}}\right) \mathbf{v}_{i}
$$

The main difficulty in computing 233 lies in the term $\frac{d \mathbf{K}_{\sigma}}{d x_{i}}$. Indeed, the $\mathbf{K}_{\sigma}$ matrix depends on the design variables $x_{i}$ and on the stress $\sigma_{i}$ of each structural element $i$, that is also depending on the design variables. A computation based on finite differences, as well as a direct computation using

$$
\frac{d \mathbf{K}_{\sigma}}{d x_{i}}=\frac{\partial \mathbf{K}_{\sigma}}{\partial x_{i}}+\frac{\partial \mathbf{K}_{\sigma}}{\partial \hat{\boldsymbol{\sigma}}} \frac{d \hat{\boldsymbol{\sigma}}}{d x_{i}},
$$

would be computationally expensive for large problems (the term $\frac{\partial \mathbf{K}_{\sigma}}{\partial \hat{\sigma}} \frac{d \hat{\boldsymbol{\sigma}}}{d x_{i}}$ requiring the solution of $N$ linear systems involving the stiffness matrices). Browne et al. (2012) proposed a direct method to compute (24), however this method is highly computational expensive, thus not tractable for large problems. Then, we apply again the adjoint method.

Following the work of Deng \& Suresh 2017) on the derivative of one buckling eigenvalue, we propose to introduce in the expression of $G_{\lambda}$ the adjoint variables

${ }_{265} \gamma_{i} \in \mathbb{R} \forall i=1, \ldots, m, \boldsymbol{\beta} \in \mathbb{R}^{6 N}$ and $\mathbf{w} \in \mathbb{R}^{3 M}$, and consider the following expression:

$G_{\lambda}:=\frac{1}{m} \sum_{i=1}^{m}\left(\mathbf{v}_{i}^{\top}\left(\mathbf{K}+c_{s} \mathbf{K}_{\sigma}\right) \mathbf{v}_{i}+\gamma_{i}\left(1-\mathbf{v}_{i}^{\top} \mathbf{K} \mathbf{v}_{i}\right)\right)+\boldsymbol{\beta}^{\top}(\hat{\boldsymbol{\sigma}}-\mathbf{Y} \mathbf{u})+\mathbf{w}^{\top}(\mathbf{f}-\mathbf{K u})$ 
where $\mathbf{Y} \in \mathbb{R}^{6 N \times 3 M}:=\sum_{i=1}^{N}\left[(\mathbf{H B})_{i}\right]$ corresponds to the block assembly of the product of the Hooke's matrix $\mathbf{H}$ and the finite element deformations matrix $\mathbf{B}$ defined for each element $i$, and $\hat{\boldsymbol{\sigma}}:=\left\{\sigma_{\mathrm{x}}^{1}, \sigma_{\mathrm{y}}^{1}, \sigma_{\mathrm{z}}^{1}, \sigma_{\mathrm{xy}}^{1}, \sigma_{\mathrm{xz}}^{1}, \sigma_{\mathrm{yz}}^{1}, \ldots, \sigma_{\mathrm{x}}^{N}, \sigma_{\mathrm{y}}^{N}\right.$, $\left.270 \sigma_{\mathrm{z}}^{N}, \sigma_{\mathrm{xy}}^{N}, \sigma_{\mathrm{xz}}^{N}, \sigma_{\mathrm{yz}}^{N}\right\}$ is the global stress component vector. Note that the two formulations of $G_{\lambda}$ in (8) and (25) are equivalent. Indeed, the eigenvectors $v_{i}$ are K-orthoganal vectors, the term $\hat{\sigma}-Y u$ corresponds to the computation of the stress components from equation (3) and the term $\mathbf{f}-\mathbf{K u}$ corresponds to equilibrium equation (2).

Differentiating 25) with respect to $x_{i}$ gives the following expression:

$$
\begin{aligned}
\frac{d G_{\lambda}}{d x_{i}}= & \frac{1}{m} \sum_{i=1}^{m}\left(2 \mathbf{v}_{i}^{\top}\left(\mathbf{K}+c_{s} \mathbf{K}_{\sigma}\right) \frac{d \mathbf{v}_{i}}{d x_{i}}+\mathbf{v}_{i}^{\top}\left(\frac{d \mathbf{K}}{d x_{i}}+c_{s} \frac{\partial \mathbf{K}_{\sigma}}{\partial x_{i}}+c_{s} \frac{\partial \mathbf{K}_{\sigma}}{\partial \hat{\boldsymbol{\sigma}}} \frac{d \hat{\boldsymbol{\sigma}}}{d x_{i}}\right) \mathbf{v}_{i}\right. \\
& \left.-\gamma_{i}\left(2 \mathbf{v}_{i}^{\top} \mathbf{K} \frac{d \mathbf{v}_{i}}{d x_{i}}+\mathbf{v}_{i}^{\top} \frac{d \mathbf{K}}{d x_{i}} \mathbf{v}_{i}\right)\right)+\boldsymbol{\beta}^{\top}\left(\frac{d \hat{\boldsymbol{\sigma}}}{d x_{i}}-\frac{d \mathbf{Y}}{d x_{i}} \mathbf{u}-\mathbf{Y} \frac{d \mathbf{u}}{d x_{i}}\right)- \\
& w^{\top}\left(\frac{d \mathbf{K}}{d x_{i}} \mathbf{u}+\mathbf{K} \frac{d \mathbf{u}}{d x_{i}}\right)
\end{aligned}
$$

Eliminating the terms $\frac{d \mathbf{v}_{i}}{d x_{i}}$ from equation (26) allows one to compute the adjoint variables $\gamma_{i} \in \mathbb{R} \forall i=1, \ldots, m$ :

$$
\begin{aligned}
& \frac{d \mathbf{v}_{i}}{d x_{i}}\left(\mathbf{K}+c_{s} \mathbf{K}_{\sigma}\right) \mathbf{v}_{i}=\gamma_{i} \frac{d \mathbf{v}_{i}}{d x_{i}} \mathbf{K v}_{i}, \forall i=1, \ldots, m \\
\Leftrightarrow & \frac{d \mathbf{v}_{i}}{d x_{i}}\left(\left(1-\gamma_{i}\right) \mathbf{K}+c_{s} \mathbf{K}_{\sigma}\right) \mathbf{v}_{i}=0, \forall i=1, \ldots, m
\end{aligned}
$$

Recalling that $\left(\mathbf{K}+\lambda_{i} \mathbf{K}_{\sigma}\right) \mathbf{v}_{i}=0, \forall i=1, \ldots, m$, the adjoint variables $\gamma_{i}, \forall i=$ $1, \ldots, m$ are chosen as:

$$
\gamma_{i}=1-\frac{c_{s}}{\lambda_{i}}, \forall i=1, \ldots, m
$$

so that equation (26) becomes:

$$
\begin{aligned}
\frac{d G_{\lambda}}{d x_{i}}= & \left.\frac{1}{m} \sum_{i=1}^{m}\left(\mathbf{v}_{i}^{\top}\left(\frac{d \mathbf{K}}{d x_{i}}+c_{s} \frac{\partial \mathbf{K}_{\sigma}}{\partial x_{i}}+c_{s} \frac{\partial \mathbf{K}_{\sigma}}{\partial \hat{\boldsymbol{\sigma}}} \frac{d \hat{\boldsymbol{\sigma}}}{d x_{i}}\right) \mathbf{v}_{i}-\gamma_{i} \mathbf{v}_{i}^{\top} \frac{d \mathbf{K}}{d x_{i}} \mathbf{v}_{i}\right)\right) \\
& +\boldsymbol{\beta}^{\top}\left(\frac{d \hat{\boldsymbol{\sigma}}}{d x_{i}}-\frac{d \mathbf{Y}}{d x_{i}} \mathbf{u}-\mathbf{Y} \frac{d \mathbf{u}}{d x_{i}}\right)-w^{\top}\left(\frac{d \mathbf{K}}{d x_{i}} \mathbf{u}+\mathbf{K} \frac{d \mathbf{u}}{d x_{i}}\right)
\end{aligned}
$$

The adjoint variable $\boldsymbol{\beta}$ is chosen in such a way that the term $\frac{d \hat{\boldsymbol{\sigma}}}{d x_{i}}$ is eliminated 
from the equation 229$)$ :

$$
\frac{c_{s}}{m} \sum_{i=1}^{m} v_{i}^{\top}\left(\frac{\partial \mathbf{K}_{\sigma}}{\partial \hat{\boldsymbol{\sigma}}} \frac{d \hat{\boldsymbol{\sigma}}}{d x_{i}}\right) v_{i}+\boldsymbol{\beta}^{\top} \frac{d \hat{\boldsymbol{\sigma}}}{d x_{i}}=0
$$

After factorization and rearrangement, the adjoint variable $\boldsymbol{\beta}$ corresponds to (Deng \& Suresh (2017); Bian \& Fang (2017)):

$$
\boldsymbol{\beta}^{\top}=-\frac{c_{s}}{m} \sum_{i=1}^{m} \mathbf{v}_{i}^{\top} \frac{\partial \mathbf{K}_{\sigma}}{\partial \hat{\boldsymbol{\sigma}}} \mathbf{v}_{i}
$$

where $\frac{\partial \mathbf{K}_{\sigma}}{\partial \hat{\boldsymbol{\sigma}}}$ corresponds to:

$$
\frac{\partial \mathbf{K}_{\sigma}}{\partial \hat{\boldsymbol{\sigma}}}=\sum_{i=1}^{N} \sum_{k=1}^{6} \frac{\partial \mathbf{K}_{\sigma}}{\partial \hat{\boldsymbol{\sigma}}_{i k}}
$$

Equation 29$)$ becomes:

$$
\begin{aligned}
\frac{d G_{\lambda}}{d x_{i}}= & \left.\frac{1}{m} \sum_{i=1}^{m}\left(\mathbf{v}_{i}^{\top}\left(\frac{d \mathbf{K}}{d x_{i}}+c_{s} \frac{\partial \mathbf{K}_{\sigma}}{\partial x_{i}}\right) \mathbf{v}_{i}-\gamma_{i} \mathbf{v}_{i}^{\top} \frac{d \mathbf{K}}{d x_{i}} \mathbf{v}_{i}\right)\right) \\
& -\boldsymbol{\beta}^{\top}\left(\frac{d \mathbf{Y}}{d x_{i}} \mathbf{u}+\mathbf{Y} \frac{d \mathbf{u}}{d x_{i}}\right)-\mathbf{w}^{\top}\left(\frac{d \mathbf{K}}{d x_{i}} \mathbf{u}+\mathbf{K} \frac{d \mathbf{u}}{d x_{i}}\right)
\end{aligned}
$$

The last adjoint variable $\mathbf{w}$ is chosen such that the term $\frac{d \mathbf{u}}{d x_{i}}$ is removed from the equation (33) :

$$
\boldsymbol{\beta}^{\top} \mathbf{Y} \frac{d \mathbf{u}}{d x_{i}}+\mathbf{w}^{\top} \mathbf{K} \frac{d \mathbf{u}}{d x_{i}}=0
$$

It is then the solution of the following system:

$$
\mathbf{K w}=-\mathbf{Y}^{\top} \boldsymbol{\beta}
$$

Finally, $\frac{d G_{\lambda}}{d x_{i}}$ becomes:

$$
\left.\frac{d G_{\lambda}}{d x_{i}}=\frac{1}{m} \sum_{i=1}^{m}\left(\mathbf{v}_{i}^{\top}\left(\frac{d \mathbf{K}}{d x_{i}}+c_{s} \frac{\partial \mathbf{K}_{\sigma}}{\partial x_{i}}\right) \mathbf{v}_{i}-\gamma_{i} \mathbf{v}_{i}^{\top} \frac{d \mathbf{K}}{d x_{i}} \mathbf{v}_{i}\right)\right)-\boldsymbol{\beta}^{\top} \frac{d \mathbf{Y}}{d x_{i}} \mathbf{u}-\mathbf{w}^{\top} \frac{d \mathbf{K}}{d x_{i}} \mathbf{u} \text { (36) }
$$

275 Thus, by solving the single additional linear system (35), the derivative (36) with respect to each design variable $x_{i}$ can be computed. This derivative corresponds to the expected variation of the value of the buckling constraint $G_{\lambda}$ in the case of removal of element $x_{i}$ from the structure. Recall that the 
topological gradient allows one to quantify the influence, on a given criterion, of

\subsection{Algorithm}

We present in the following our algorithm for structural topological optimization. It follows the general idea of topological gradient-based algorithms, that is to iteratively remove a fraction of the volume of the design domain on the basis of the values of topological gradients (see subsection 4.1). The specific character of the algorithm is in the way the gradients of stress and buckling constraints are computed and combined, as well as in the criteria considered to iteratively update the topology of the structure. In order to build a single topological gradient field $T_{w}$, we follow the approach consisting in defining a weighted sum of the considered fields (Suresh et al. (2012)). For each element $x_{i}$, we propose to combine the values of $T_{\sigma}$ and $T_{\lambda}$ through the following weighted sum:

$$
T_{w}\left(x_{i}\right)=T_{\sigma}\left(x_{i}\right)+\left(\frac{c_{s}}{\lambda_{c r}}\right)^{2} T_{\lambda}\left(x_{i}\right)
$$

where $\lambda_{c r}$ is the critical buckling load. The factor $\left(c_{s} / \lambda_{c r}\right)^{2}$ allows us to control the weight of $T_{\lambda}$. If the value of $c_{s} / \lambda_{c r}$ is close to one, then the optimization is driven by both stress and buckling topological gradients; if $\lambda_{c r}^{2}$ is much larger than $c_{s}$, i.e., the structure features a high resistance to buckling, only $T_{\sigma}$ controls the optimization. Along the iterative process, the topology of the structure is updated following the approach presented in subsection 4.1, where the considered topological gradient is $T_{w}$. 
The topology is updated at each iteration in such a way that the problem constraints are satisfied. This ensures that a feasible solution is always computed. One can however observe (Deng \& Suresh $(2015))$ that removing fractions $\Delta \alpha$ of volume may lead to a divergence of the optimization process. Hence, we propose a further condition to be satisfied at each iteration in order to update the topology by removing $\Delta \alpha$. This condition is based on the (relative) variation of the value of the stress constraint $G_{\sigma}$ between the one associated to the current structure, $\boldsymbol{\Omega}$, and the one associated to the perturbed one, $\boldsymbol{\Omega}^{\tau}$ :

$$
\frac{\left|G_{\sigma}(\boldsymbol{\Omega})-G_{\sigma}\left(\boldsymbol{\Omega}^{\tau}\right)\right|}{\left|G_{\sigma}(\boldsymbol{\Omega})\right|} \leq \overline{\Delta G_{\sigma}}
$$
of the meshed domain, assuming that such a value is zero for the elements which have been removed. Following (Krishnakumar \& Suresh (2015)), the extrapolation process consists first in evaluating, for each node of the mesh, the mean of the topological gradients $T_{\sigma}$ of the elements connected to such 315 a node, and assigning this value to the node. Then, for each element of the 
meshed domain, the value of $T_{\sigma}$ is recomputed by averaging the values assigned to its neighboring nodes. Note that this procedure leads to a smoothing of the topological gradients. This smoothing is usually referred to as a filtering scheme (Bendsoe \& Sigmund (2013)) and allows one to prevent the checkerboard effect (Sigmund \& Petersson (1998)).

The proposed algorithm is presented in Algorithm 2 Note that the procedure to compute multiple buckling modes, presented in Sect.III, is nested in Algorithm 2 (steps 3 and 7).

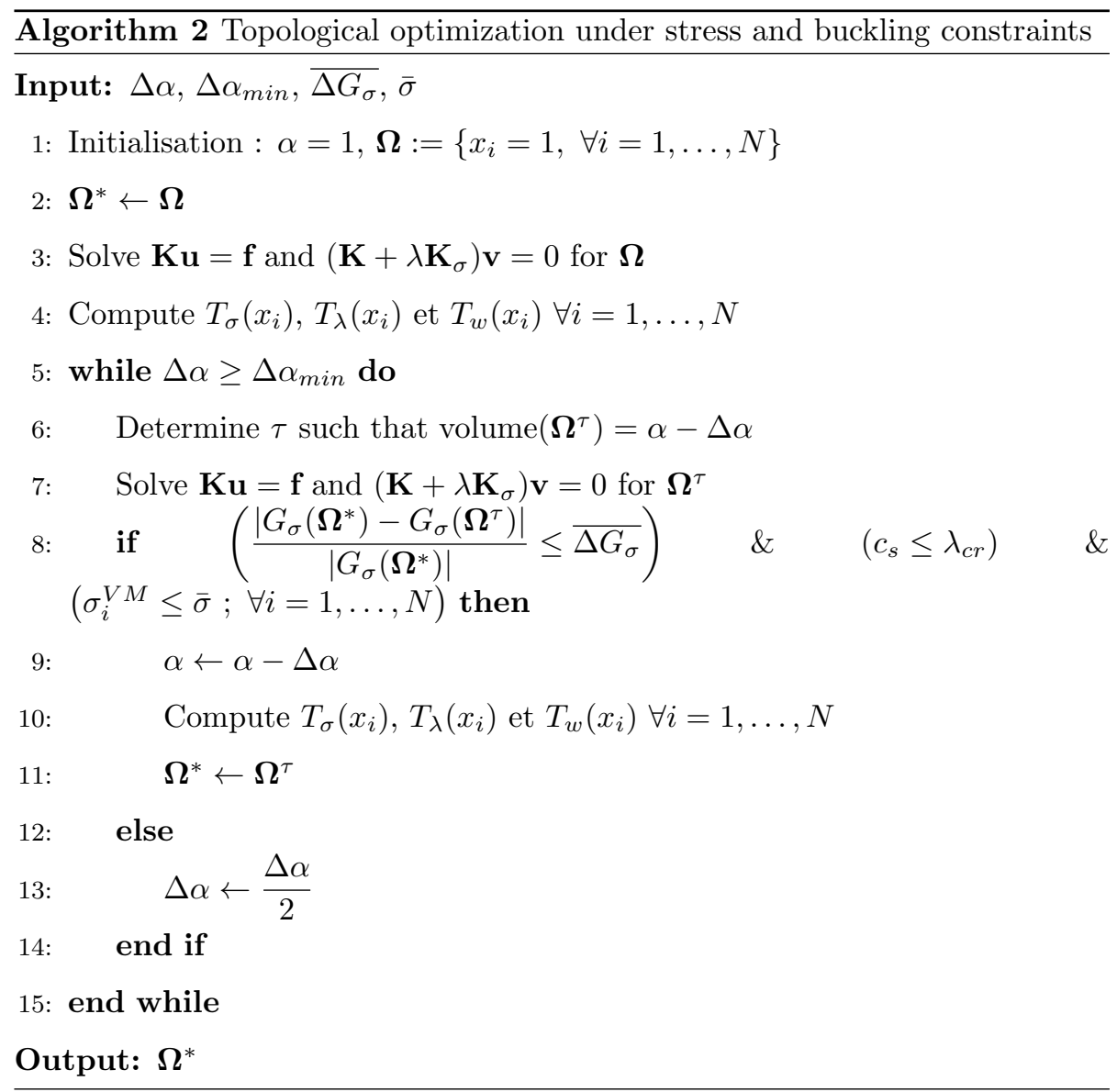




\section{Numerical results}

325 In this section, we first analyze the performance of Algorithm 1 to compute a set of eigenvalues. Then, numerical results for topological optimization applications, obtained performing Algorithm 2, are illustrated and discussed.

In all numerical experiments in this section, the material considered is an aluminium with a Young modulus equals to $72000 \mathrm{MPa}$ and a Poisson ratio equals to 0.33 . The density of aluminum is $0.00285 \mathrm{~g} . \mathrm{mm}^{-3}$. The value of the limit stress $\bar{\sigma}$ is $510 \mathrm{MPa}$. The safety coefficient $c_{s}$ is fixed to 1 . Recall that we consider three-dimensional cubic elements to discretize the design domain of the considered structures.

From numerical experiments, we set the parameter $P$ to 4 in the stress constraint (5), and the $\overline{\Delta G_{\sigma}}$ stress threshold is set to 0.01 . The material fraction $\Delta \alpha$ is initialized to 0.1 . The threshold $\Delta \alpha_{\min }$ is fixed at a value of 1 divided by the number of elements resulting from the discretization of the design domain. This ratio corresponds to the volume occupied by an element in the initial structure. Thus, the solution algorithm stops when it is no longer possible to remove an element from the structure.

The proposed algorithms have been implemented in Fortran and parallelized by using OpenMP commands. In Algorithm 1 the tolerance $\epsilon$ is set to $10^{-4}$. The codes were run on a 32-bit Linux OS computer with an Intel(R) Core(TM) i7-4710MQ CPU @ 2.50GHz and 16GB RAM.

\subsection{Buckling analysis results}

Let us first consider the computation of a set of buckling modes on the L-structure illustrated in Figure 2, through Algorithm 1. The hatched area corresponds to the foundation, i.e. clampled displacements. A load F is uniformly distributed over the red area in Figure 2 and is equal to 35,000 N. The structure is discretized with cubes of $1 \mathrm{~mm}$ in length, leading to 331776 finite elements and 364429 nodes.

We consider the computation of the first 3 buckling eigenvalues (i.e., $m=3$ in Algorithm 1) associated to the considered structure. On the one hand, we 
focus on the importance of considering the dimension $\widetilde{m}$ of the set $\mathbf{V}$ large enough to compute the first 3 buckling eigenvalues. On the other hand, we investigate the impact of the dimension $\widetilde{m}$ of $\mathbf{V}$ on the computational cost.

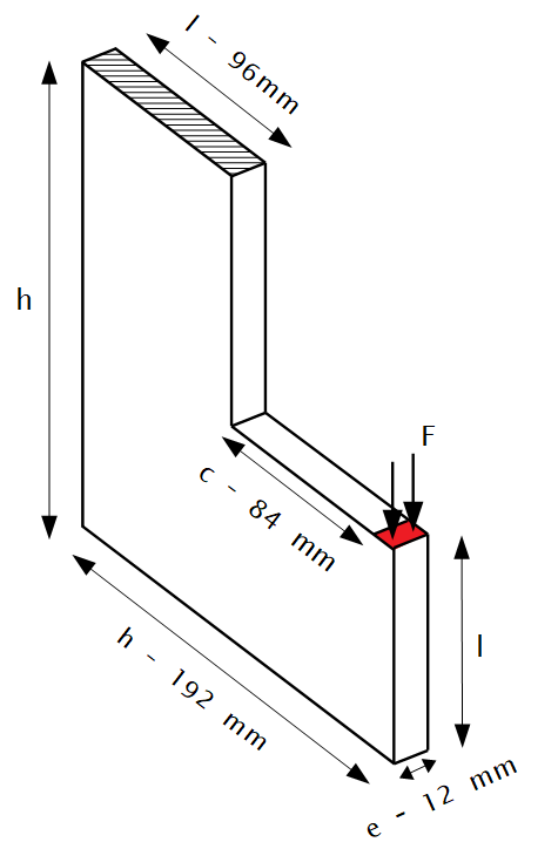

Figure 2: L-shaped structure

Let us recall that, through Algorithm 1, the eigenvalues are determined in ascending order according to their modulus. Thus, the first three eigenvalues obtained may not correspond to positive eigenvalues. In Table 1, we report the eigenvalues obtained for values of $\widetilde{m}$ varying from three to five.

Table 1: L-shaped structure: Eigenvalues obtained for different dimensions of the set $\mathbf{V}$

\begin{tabular}{|c|c|c|c|c|c|}
\hline $\begin{array}{c}\text { Dimension } \widetilde{m} \\
\text { of the set } \mathbf{V}\end{array}$ & $\lambda_{1}$ & $\lambda_{2}$ & $\lambda_{3}$ & $\lambda_{4}$ & $\lambda_{5}$ \\
\hline 3 & -2.222 & 2.677 & 7.038 & NA & NA \\
\hline 4 & -2.222 & 2.677 & 7.038 & -7.577 & NA \\
\hline 5 & -2.222 & 2.677 & 7.038 & -7.577 & 11.352 \\
\hline
\end{tabular}


For the structure under study, we note that with $\widetilde{m}$ equals to three or four, we are not able to compute the first 3 positive eigenvalues. In the case of $\widetilde{m}$ equals to five, we observe that the three smallest positive eigenvalues correspond to the second, third and fifth eigenvalues of smallest modulus respectively. Thus, the dimension $\widetilde{m}$ of $\mathbf{V}$ must be greater than or equal to five in order to compute the first three positive eigenvalues. It is important to note that, along a process of structural optimization, the structural topology changes at each iteration. Thus, the buckling eigenvalues may switch their positions along the process. Therefore, the dimension $\widetilde{m}$ of $\mathbf{V}$ must be large enough to ensure that all the eigenvalues to be searched for are obtained at each iteration of the optimization process. However, if the dimension $\widetilde{m}$ of $\mathbf{V}$ is too large, then the buckling analysis may be highly time consuming.

We now investigate the impact of the dimension $\widetilde{m}$ of $\mathbf{V}$ on the computational cost. For different values of $\widetilde{m}$, we report in Table 2 the computing time, the number of iterations, and the number of $\mathbf{K} \mathbf{v}$ and $\mathbf{K}_{\sigma} \mathbf{v}$ products evaluated to converge to the seeked buckling eigenvalues.

Table 2: L-shaped structure: Computing time according to the size of the eigenvalue search space

\begin{tabular}{|c|c|c|c|c|c|c|}
\hline $\begin{array}{l}\text { Dimension } \widetilde{m} \\
\text { of the set } V\end{array}$ & Computing time & $\begin{array}{l}\text { Number of } \\
\text { iterations }\end{array}$ & $\begin{array}{c}\text { Number of } \\
\text { products } \mathbf{K v}\end{array}$ & $\begin{array}{l}\text { Computing time } \\
\text { of one product } \mathbf{~ K v}\end{array}$ & $\begin{array}{c}\text { Number of } \\
\text { products } \mathbf{K}_{\sigma} \mathbf{v}\end{array}$ & $\begin{array}{l}\text { Computing time } \\
\text { of one product } \mathbf{K}_{\sigma} \mathbf{v}\end{array}$ \\
\hline 5 & $49 \min 3 \mathrm{~s}$ & 33 & 27777 & \multirow{5}{*}{$0.135 \mathrm{~s}$} & 506 & \multirow{5}{*}{$0.98 \mathrm{~s}$} \\
\hline 6 & $31 \mathrm{~min} 21 \mathrm{~s}$ & 18 & 28099 & & 328 & \\
\hline 8 & $38 \min 47 \mathrm{~s}$ & 17 & 39117 & & 428 & \\
\hline 9 & $44 \mathrm{~min} 21 \mathrm{~s}$ & 15 & 41043 & & 422 & \\
\hline 10 & $49 \min 51 \mathrm{~s}$ & 15 & 45089 & & 463 & \\
\hline
\end{tabular}

We note that the product $\mathbf{K}_{\sigma} \mathbf{v}$ is on average 7 times more expensive than the product $\mathbf{K v}$. The operation $\mathbf{K}_{\sigma} \mathbf{v}$ leads moreover to a larger memory footprint than $\mathbf{K v}$, since each geometric stiffness matrix element depends on its own stress matrix (6) (while $\mathbf{K}$ is constituted by equal blocks). Through the proposed method, the number of $\mathbf{K}_{\sigma} \mathbf{v}$ operations is kept low, thus yielding to important savings in time and memory consumption. This crucial point about memory consumption already led the authors in Yadav \& Suresh (2014) to develop an 
alternative approach to what is commonly done in standard commercial software (such as ANSYS - Stolarski et al. (2018)), where sparse direct solvers may require huge memory consumption. In such a case, the authors recall that, as an example, for a matrix with a size of 1 million, $1 \mathrm{~GB}$ of memory is needed to store the stiffness matrix and 10 to $20 \mathrm{~GB}$ additional memory for the factorization. The method that we propose follows a similar approach than the one in Yadav

390 \& Suresh (2014) for efficient memory handling, with the additional advantage to be able to compute multiple eigenvalues.

Comparing the results obtained for $\widetilde{m}$ equals to 5 and 6 , we observe a reduction of the computing time by a factor of 1.56 , although the number of $\mathbf{K v}$ products remains stable. The number of iterations and the number of $\mathbf{K}_{\sigma} \mathbf{v}$ products decrease when the dimension of the search space is increased. Nevertheless, for larger values of $\widetilde{m}$, computing time increases due to a larger number of $\mathbf{K v}$ products, although the number of iterations to converge decreases.

We conclude that the choice of the value of $\widetilde{m}$ of $\mathbf{V}$ in Algorithm 1 should try to meet the right trade-off between computing time saving and the need to capture every proper buckling value required throughout the optimization process.

\subsection{Topological optimization results}

In this section, we present numerical results to validate our topology optimization approach and to illustrate its efficiency on real mechanical structures. We consider two different mechanical structures: a beam under a compressive load, and a real application arising in the aeronautic field. The first application additionally allows us to highlight the interest of considering multiple buckling eigenvectors in the optimization process, while the second shows the importance of buckling constraints on the optimization process.

\subsubsection{Beam under a compressive load}

The first numerical example of topological optimization is a structure under a compressive load illustrated in Figure 3 . The dimensions of the structure are 
$l$, equals to $240 \mathrm{~mm}, h$, equals to $42 \mathrm{~mm}$ and $e$, equals to $6 \mathrm{~mm}$. The load $F$ is $18,360 \mathrm{~N}$ and is applied uniformly over the red area in Figure 3. The hatched area corresponds to the fixed area. The structure is discretized with cubes with a length of $2 \mathrm{~mm}$, leading to 52920 elements. We report the results obtained when solving problem $(\mathrm{P})$ by applying the proposed topological optimization algorithm. Table 3 gives, in terms of the considered number of buckling modes, the optimal mass (i.e., the value of the objective function of $(\mathrm{P})$ ), the final crit${ }_{420}$ ical buckling load, the final maximum Von Mises stresses $\left(\max _{i=1, \ldots, N} \sigma_{i}^{V M}\left(x^{*}\right)\right)$ and the computing time.

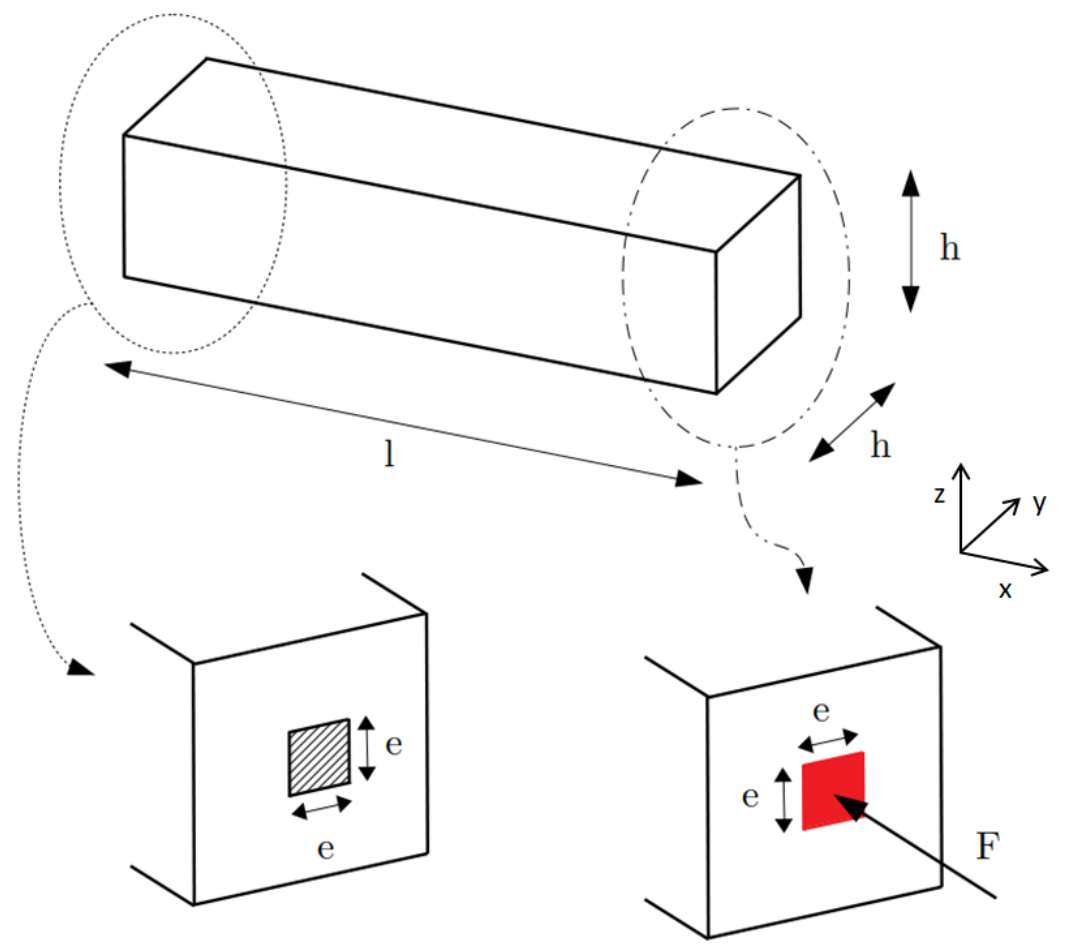

Figure 3: Beam under a compressive load: Design space 
Table 3: Beam under a compressive load: Mass, Critical buckling load, Maximal Von Mises stress and Computing time

\begin{tabular}{|c|c|c|c|c|}
\hline $\begin{array}{c}\text { Number of considered } \\
\text { buckling modes }\end{array}$ & Mass (g) & $\begin{array}{c}\text { Critical buckling } \\
\text { load }\end{array}$ & $\begin{array}{c}\text { Maximal Von Mises } \\
\text { stress }\end{array}$ & Computing time \\
\hline 1 & 178.50 & 1.009 & 299.25 & $3 \mathrm{~h} 45 \mathrm{~min}$ \\
\hline 4 & 148.17 & 1,07 & 304.94 & $5 \mathrm{~h} 38 \mathrm{~min}$ \\
\hline 6 & 149.44 & 1.0001 & 265.04 & $8 \mathrm{~h} 55 \mathrm{~min}$ \\
\hline
\end{tabular}

Figures 4 and 5 illustrate the evolution, with respect to the number of computed buckling eigenvectors, of the structural mass and the critical buckling load respectively. As regards the structural mass, when the optimization is run taking into account only the first buckling eigenvector, the optimized structure has a mass of $178.50 \mathrm{~g}$ (Figure 6(a)). A reduction of $17 \%$ is observed when the first four buckling modes are considered (Figure 7 (a)). From Figure 5 the evolution of the critical buckling load when only one buckling mode is considered, appears erratic. This is characteristic of buckling mode-switching behaviour. A smoother evolution of the critical buckling load is observed when multiple buckling modes are considered. This is related to a reduced mode-switching behaviour along the optimization process.

Whatever the number of buckling modes considered, the proposed algorithm always converges towards an optimized structure. In the specific case of a beam in compression, the optimized structures, and therefore their masses, change according to the number of buckling modes considered in the constraint [8]. From a mechanical point of view, this influence of buckling modes can be explained by comparing the two obtained structures (Figure 6(b) and Figure 7(b)). Indeed, in the case of a beam in compression, its resistance to buckling is characterized by its moment of inertia with respect to the $y$ and $\mathrm{z}$ axes. For the case of four buckling modes, we observe that the material is mostly placed in the corners of the design space and the legs are connected each other by stiffeners in order to counter the effect of the first buckling mode, generating a better buckling resistance compared to the structure obtained with only one buckling eigenvec- 
tor. Note that this solution is very interesting from a point of view of structural engineering, that would add these stiffeners in a post-processing phase if they were not automatically generated by the optimization algorithm. In addition, when four buckling modes are considered, the critical buckling load decreases less rapidly than in the case of one buckling mode, allowing a better layout of the material within the structure and generating a lighter structure. Finally, comparing the plots of the Von Mises stress in Figures 6(d) and 7(e), one can note that the stress values are more homogeneous for the structure optimized by considering 4 buckling modes than the values obtained considering only one buckling mode.

When six buckling modes are considered, the mass of the obtained optimal structure has a value of $149.44 \mathrm{~g}$, that is very close to that obtained in the case of four modes. Moreover, one can note that the distributions of the Von Mises stress in Figures $7(\mathrm{e})$ and $8(\mathrm{f})$ are also very close. Comparing the structures obtained in the two cases, displayed respectively in Figures 7(a) and 8(a), one can remark that they differ with respect to the points where the loads are applied. These structures correspond to different local optima.

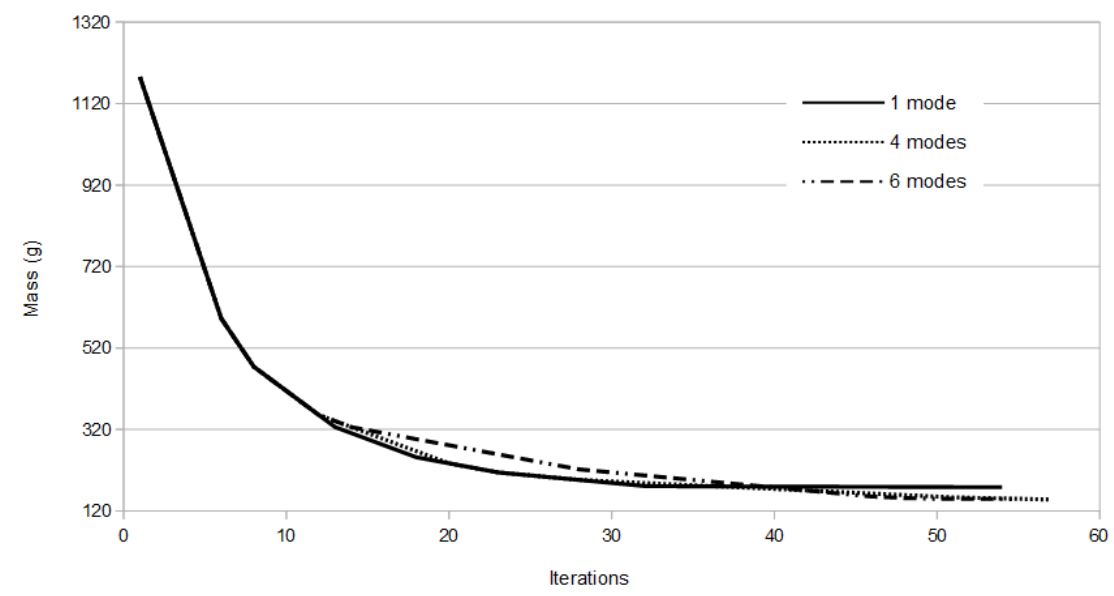

Figure 4: Beam under a compressive load: Evolution of the mass according to the number of buckling modes considered 


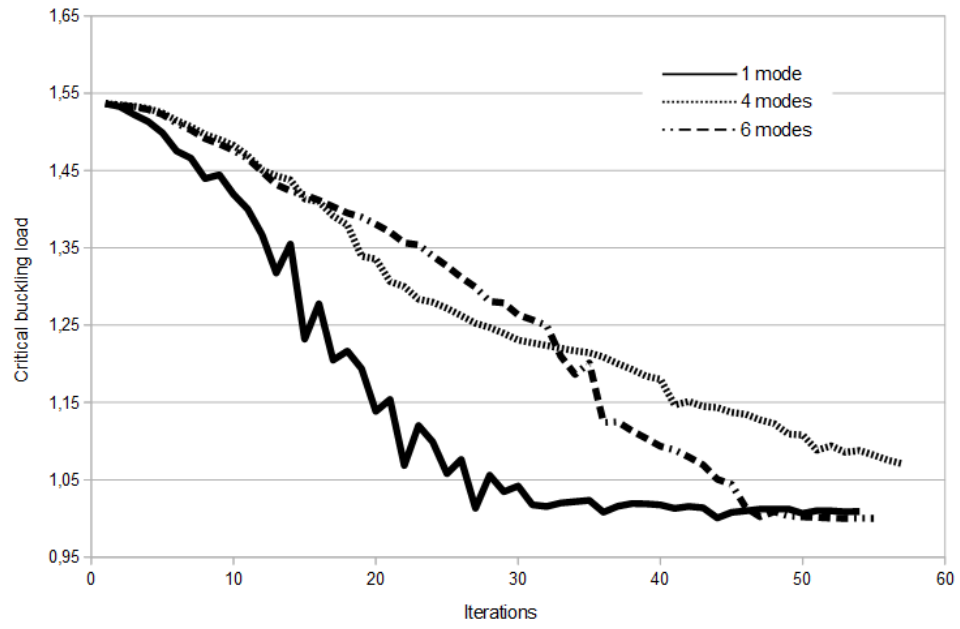

Figure 5: Beam under a compressive load: Evolution of the critical buckling load according to the number of buckling modes considered 


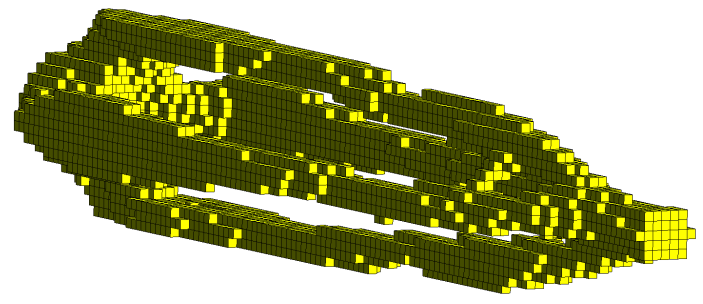

(a) Optimized structure

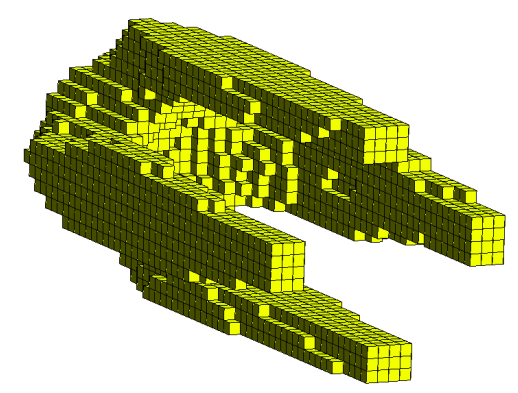

(b) Cutting plane according to the $y$ and $z$ axes

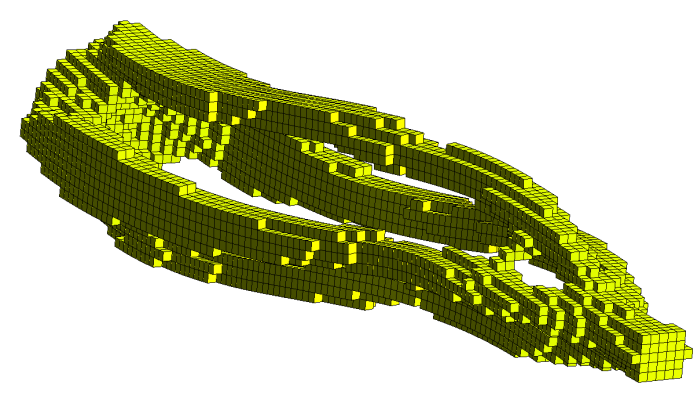

(c) First buckling mode

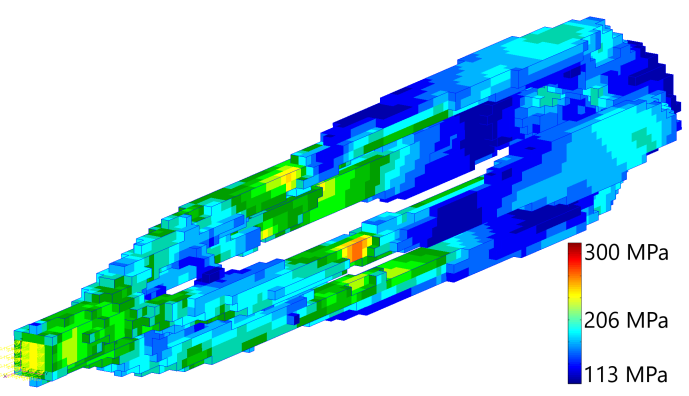

(d) Von Mises Stress

Figure 6: Beam under a compressive load: Structure obtained with 1 buckling mode 


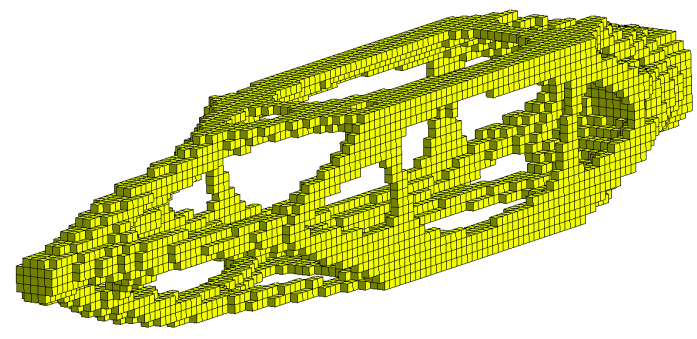

(a) Optimized structure
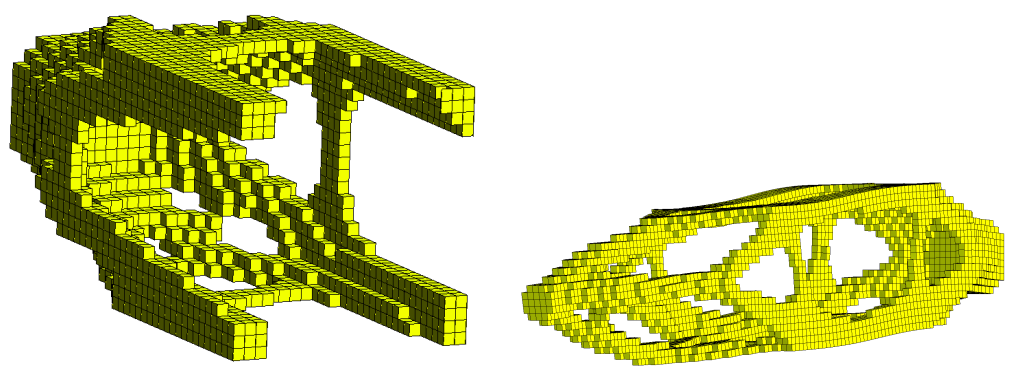

(b) Cutting plane according to the $y$

(c) First and second buckling mode and $z$ axes

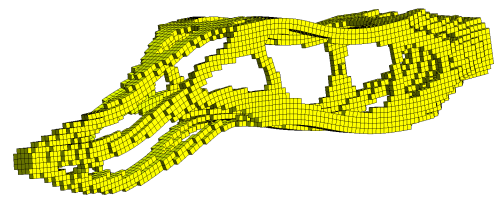

(d) Third and fourth buckling mode

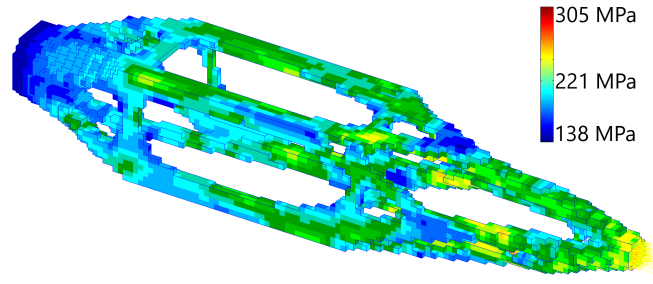

(e) Von Mises Stress

Figure 7: Beam under a compressive load: Structure obtained with 4 buckling modes 


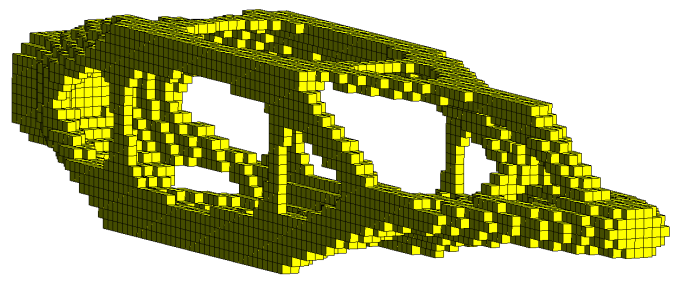

(a) Optimized structure
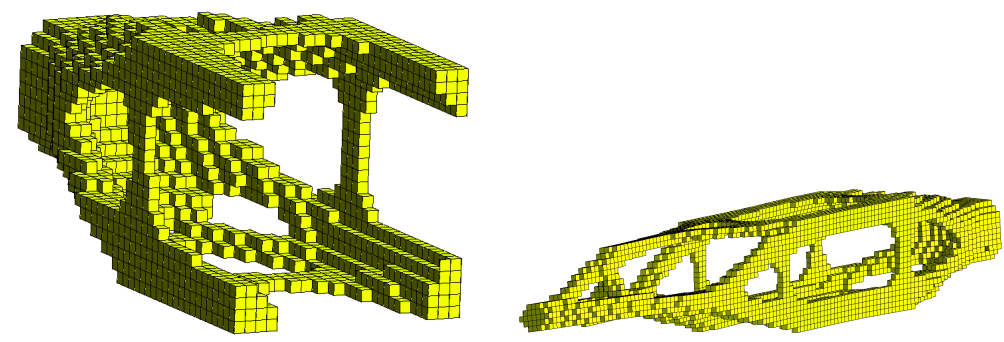

(b) Cutting plane according to the $y$

(c) First and second buckling mode and $z$ axes

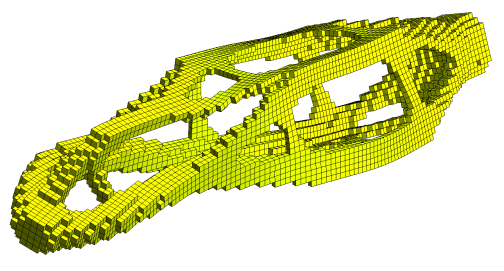

(d) Third and fourth buckling mode

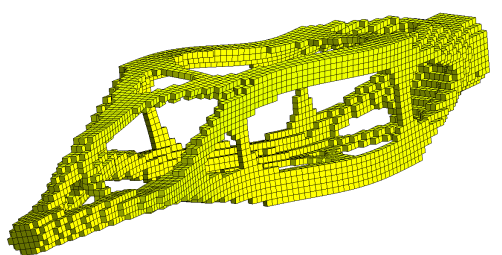

(e) Fifth and sixth buckling mode

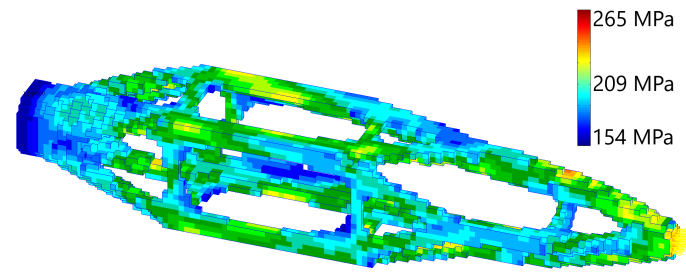

(f) Von Mises Stress

Figure 8: Beam under a compressive load: Structure obtained with 6 buckling modes 


\subsubsection{Gooseneck - aeronautical structure}

The second numerical example is an aeronautical application (illustrated in Figure 9p, referred to as gooseneck. Several structures of this type appear on the landing gear doors of an aircraft. The area in red corresponds to a load of -12 $128 \mathrm{~N}$ along the $\mathrm{x}$ axis, $-35 \mathrm{~N}$ along the y axis, and $3456 \mathrm{~N}$ along the $\mathrm{z}$ axis. The hatched area represents the fixed area. The structure is discretized by cubic finite elements with a length of $4 \mathrm{~mm}$, leading to 31670 elements. In this example, numerical experiments show that considering more than one buckling mode does not modify the final optimal solution. This can also be explained from a mechanical point of view, considering the dimensions of the structure and the direction of the applied loads. Thus, taking into account that the optimization process for this structure may be associated to high computing times, we limit the optimization to one buckling mode. We compare the optimal structure that is obtained with and without buckling constraint, to highlight the important role of the buckling constraint on the the optimal structural design.

Table 4 reports the optimal structural mass, the final critical buckling loads, and the maximal Von Mises stresses, obtained in the two cases corresponding to the optimization carried out with and without considering buckling constraints. 


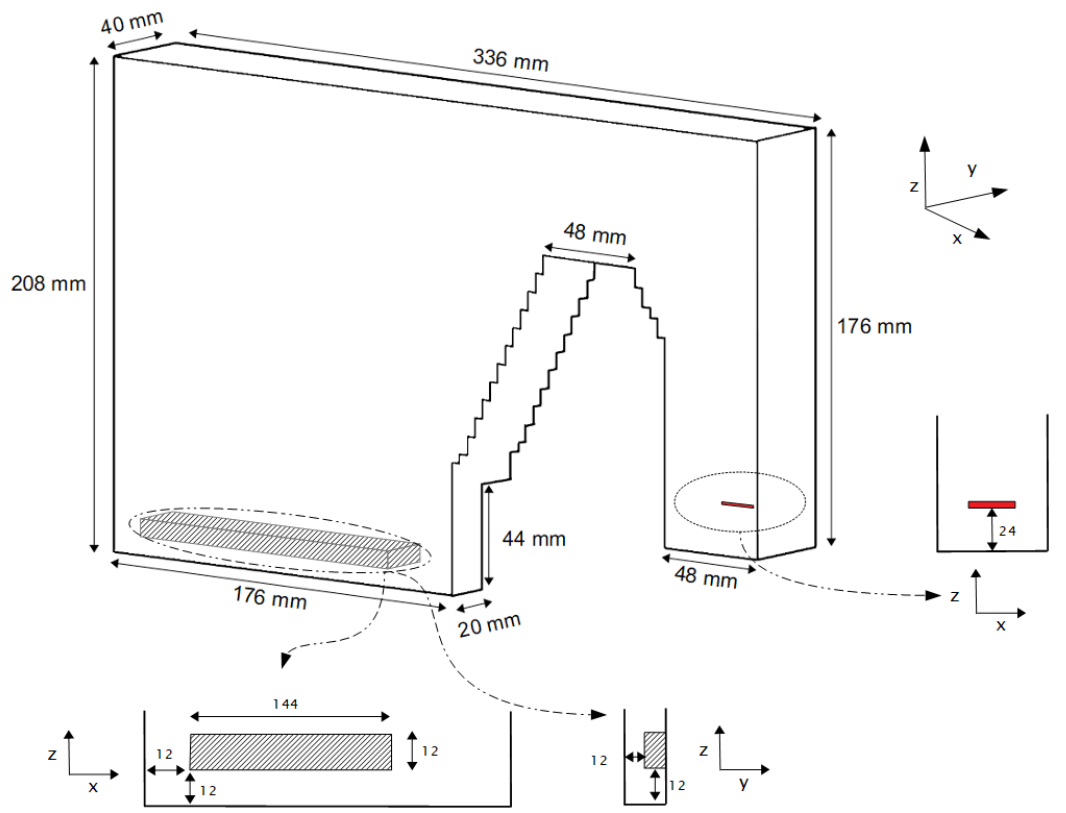

Figure 9: Gooseneck: Design space of the structure

Table 4: Gooseneck structure: Optimization results with and without buckling constraint

\begin{tabular}{|c|c|c|c|c|}
\hline $\begin{array}{c}\text { Number of considered } \\
\text { buckling eigenvectors }\end{array}$ & Mass (g) & $\begin{array}{c}\text { Critical buckling } \\
\text { load }\end{array}$ & $\begin{array}{c}\text { Maximal Von } \\
\text { Mises stress }\end{array}$ & Computational time \\
\hline 0 & 199.52 & 0.743 & 497.44 & $30 \mathrm{~min}$ \\
\hline 1 & 210.05 & 1.07 & 469.18 & $7 \mathrm{~h} \mathrm{23min}$ \\
\hline
\end{tabular}

Optimization without buckling constraint leads to a structure (Figure 11) with a mass of $199.52 \mathrm{~g}$, which does not resist to buckling as the critical load is equal to 0.743 . Taking into account the buckling constraint in the optimization problem leads to an optimized structure (Figure 12) with a mass of $210.05 \mathrm{~g}$, corresponding to an increase of $5.2 \%$.

485

In Figure 10, we illustrate the evolution of the critical buckling load during the optimization. It can be seen that in the first iterations the values of the critical buckling load are very high. For the initial structure, the buckling analysis leads to a critical buckling load of 277.97. Recall that in the optimization al- 
gorithm the global topological gradient $T_{w}$ is a weighted sum of the topological gradients $T_{\sigma}$ and $T_{\lambda}$. In particular, the topological gradient associated with the buckling constraint, $T_{\lambda}$, is multiplied by the inverse of the square of the critical load (see (37)). Thus, for this application, at the initial iterations only the topological gradient $T_{\sigma}$ related to the stress constraints drives the optimization process. This explains the similarity of the structures obtained in Figures 11 495 and 12

The major difference is in the buckling load: its value associated to the later structure is greater than one, meaning that this structure is not subject to buckling failure. The first and the second buckling modes for the structures obtained respectively without buckling constraints and with such constraints are illustrated in Figure 11(c)-(d)-(e) and Figure 12(c)-(d).

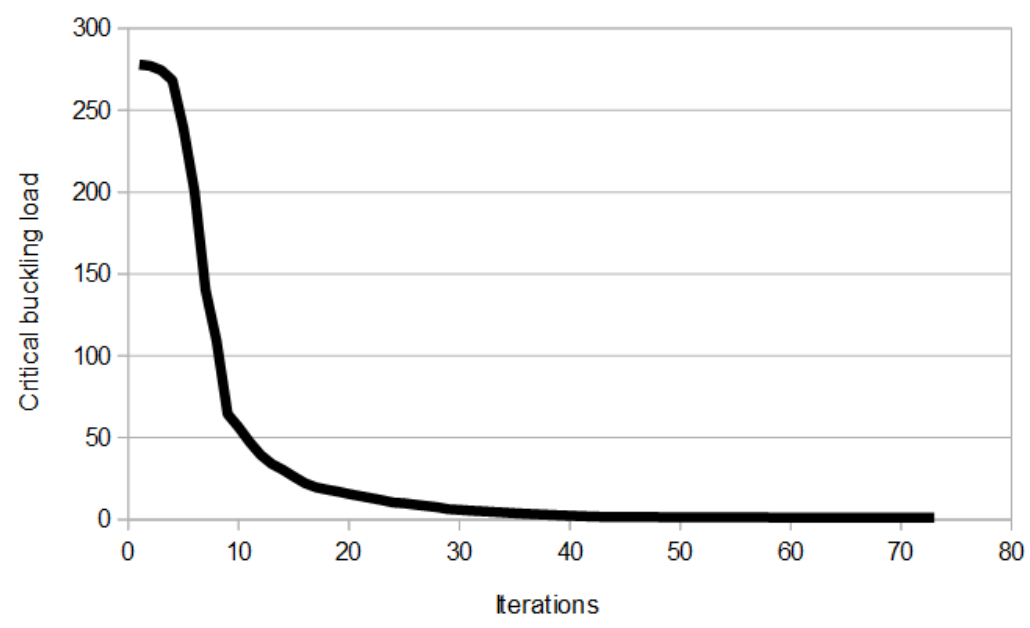

Figure 10: Gooseneck structure: Evolution of the critical buckling load 


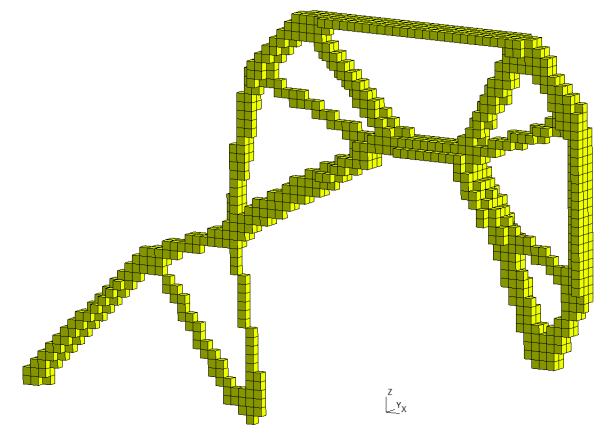

(a) Optimal structure

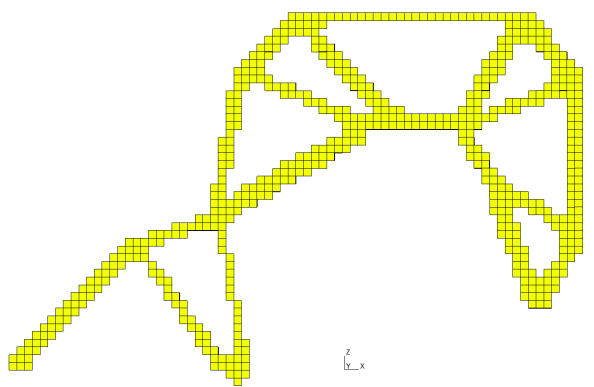

(b) Side view of the optimal structure

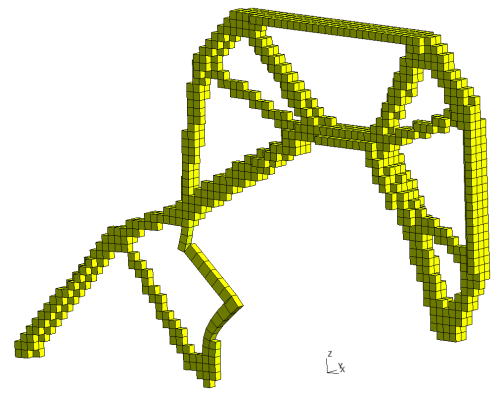

(c) First buckling mode
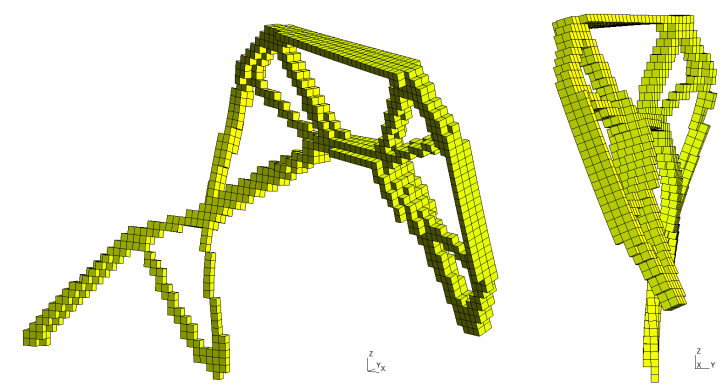

(d) Second buckling mode - First view (e)

Second

buckling mode -

Second view

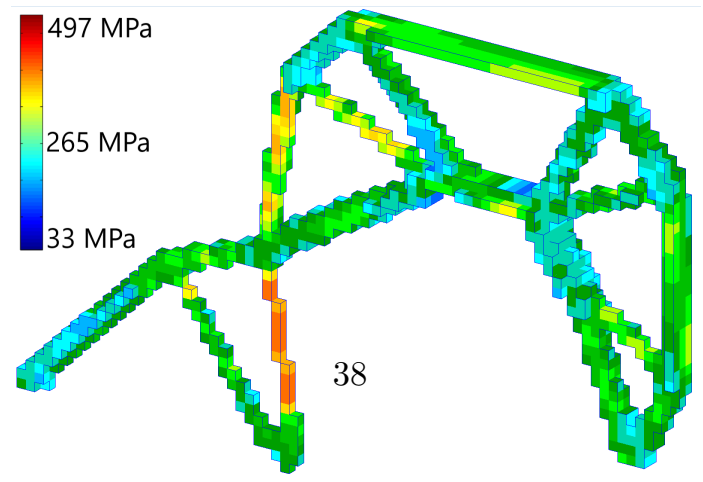

(f) Von Mises Stress

Figure 11: Gooseneck: Structure obtained without buckling constraint 


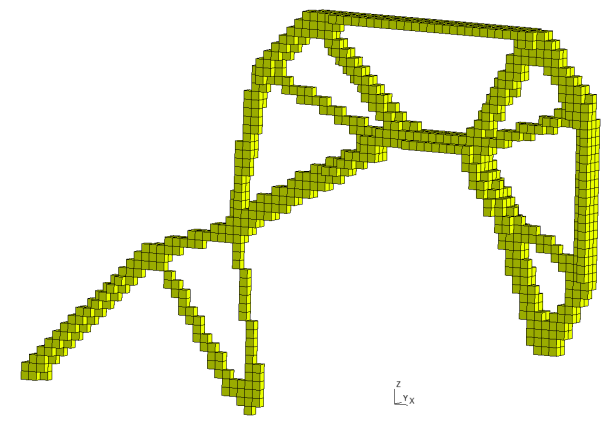

(a) Optimal structure
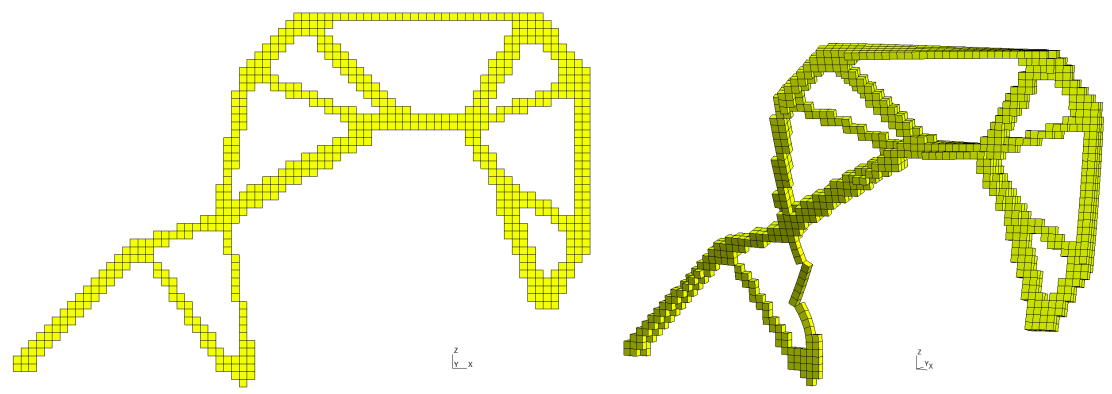

(b) Side view of the optimal structure

(c) Buckling mode of the optimal structure - First view
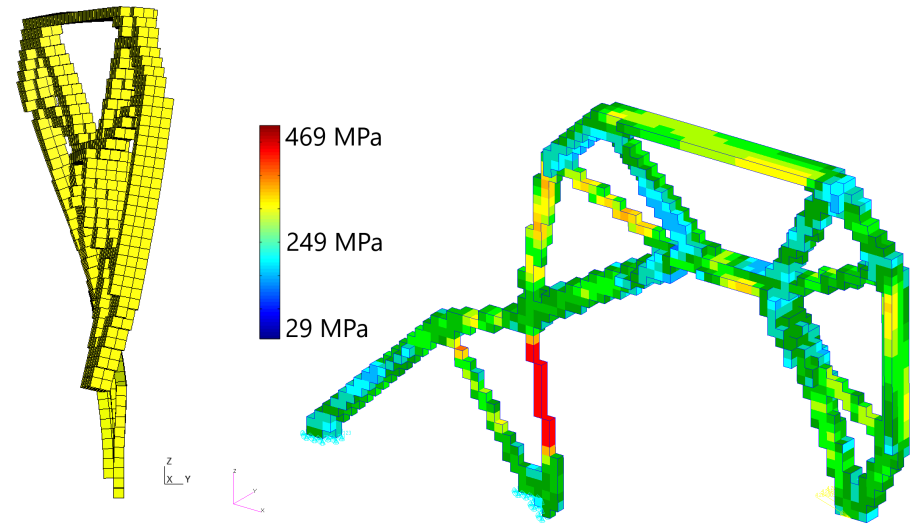

(d) Buckling

(e) Von Mises Stress

mode of the op-

timal structure

- Second view

Figure 12: Gooseneck: Structure obtained with buckling constraint 


\section{Conclusion}

We address the topological optimization of continuum structures, whose mass is to be minimized, subject to stress and to buckling constraints.

We propose, for the buckling analysis, a variant of the inverse-iterations algo-

rithm that is devised to compute a set of eigenvalues. It features low memory requirements, thus allowing one to handle large-scale problems, that are not uncommon in the context of topology optimization, specially when 3D discretizations are considered.

To optimize the topology of structures under stress and buckling constraints, we develop an algorithm that follows a topological gradients-based approach. We use aggregation formulations for the two considered constraints, and apply the adjoint method to efficiently compute their gradients. The proposed algorithm allows one to handle optimization of structures subject to constraints that are attracting an increasing attention in the structural engineering community. It relies on our proposed buckling analysis and gradient computation, that are embedded in a simple scheme to iteratively compute the optimized topology for the targeted structure. Numerical results on mechanical structures, including a real application arising in the aeronautical field, show the effectiveness of the proposed optimization algorithm.

The proposed topology optimization algorithm features a flexibility allowing one to easily implement further constraints, e.g., the compliance. We consider this as a perspective for future work. Moreover, the inclusion of additive manufacturing constraints, such as the trajectory of the deposition of the material in the case of the Wire Arc Additive Manufacturing method (Radel et al. (2019)), will be investigated.

\section{Acknowledgement}

The authors gratefully acknowledge the support by AVANTIS Project and the French National Association of Reasearch and Technology (ANRT). 


\section{References}

530 Allaire, G. (2015). A review of adjoint methods for sensitivity analysis, uncertainty quantification and optimization in numerical codes. Ingénieurs de l'Automobile, 836, 33-36.

Allaire, G., Jouve, F., \& Toader, A.-M. (2004). Structural optimization using sensitivity analysis and a level-set method. Journal of computational physics, 194(1), 363-393.

Anderson, E., Bai, Z., Bischof, C., Blackford, L. S., Demmel, J., Dongarra, J., Du Croz, J., Greenbaum, A., Hammarling, S., McKenney, A. et al. (1999). LAPACK Users' guide. SIAM.

Bendsoe, M. P. \& Sigmund, O. (2013). Topology optimization: theory, methods, and applications. Springer Science \& Business Media.

Bian, X., \& Fang, Z. (2017). Large-scale buckling-constrained topology optimization based on assembly-free finite element analysis. Advances in Mechanical Engineering, 9, 1687814017715422.

Bian, X., Yadav, P., \& Suresh, K. (2015). Assembly-free buckling analysis for topology optimization. In ASME-IDETC Conference, Boston, MA.

Browne, P., Budd, C., Gould, N., Kim, H., \& Scott, J. (2012). A fast method for binary programming using first-order derivatives, with application to topology optimization with buckling constraints. International Journal for Numerical Methods in Engineering, 92, 1026-1043.

Bruyneel, M., Colson, B., \& Remouchamps, A. (2008). Discussion on some convergence problems in buckling optimisation. Structural and Multidisciplinary Optimization, 35, 181-186.

Calvel, S. (2004). Conception d'organes automobiles par optimisation topologique. Ph.D. thesis Université Paul Sabatier-Toulouse III. 
Céa, J., Garreau, S., Guillaume, P., \& Masmoudi, M. (2000). The shape and topological optimizations connection. Computer methods in applied mechanics and engineering, 188, 713-726.

Choi, K. K., \& Kim, N.-H. (2006). Structural sensitivity analysis and optimization 1: linear systems. Springer Science \& Business Media.

${ }_{560}$ Cook, R. D., Malkus, D. S., Plesha, M. E., \& Witt, R. J. (1974). Concepts and applications of finite element analysis volume 4. Wiley New York.

Costa, G. \& Montemurro, M. (2020). Eigen-frequencies and harmonic responses in topology optimisation: A cad-compatible algorithm. Engineering Structures, 214, 110602.

565 Costa, G., Montemurro, M., \& Pailhès, J. (2018). A 2d topology optimisation algorithm in nurbs framework with geometric constraints. International Journal of Mechanics and Materials in Design, 14(4), 669-696.

Costa, G., Montemurro, M., \& Pailhès, J. (2019a). Minimum length scale control in a nurbs-based simp method. Computer Methods in Applied Mechanics and Engineering, 354, 963-989.

Costa, G., Montemurro, M., \& Pailhès, J. (2019b). Nurbs hyper-surfaces for 3d topology optimization problems. Mechanics of Advanced Materials and Structures, (pp. 1-20).

Costa, G., Montemurro, M., Pailhès, J., \& Perry, N. (2019c). Maximum length scale requirement in a topology optimisation method based on nurbs hypersurfaces. CIRP Annals, 68(1), 153-156.

Deaton, J. D. \& Grandhi, R. V. (2014). A survey of structural and multidisciplinary continuum topology optimization: post 2000. Structural and Multidisciplinary Optimization, 49(1), 1-38.

Deng, S., \& Suresh, K. (2015). Multi-constrained topology optimization via the topological sensitivity. Structural and Multidisciplinary Optimization, 51, 987-1001. 
Deng, S. \& Suresh, K. (2017). Topology optimization under thermo-elastic buckling. Structural and Multidisciplinary Optimization, 55(5), 1759-1772.

Dunning, P. D., Ovtchinnikov, E., Scott, J., \& Kim, H. A. (2016). Levelset topology optimization with many linear buckling constraints using an efficient and robust eigensolver. International Journal for Numerical Methods in Engineering, 107, 1029-1053.

Duysinx, P., \& Sigmund, O. (1998). New developments in handling stress constraints in optimal material distribution. In Proc of the 7th AIAA/USAF/NASAISSMO Symp on Multidisciplinary Analysis and Optimization (pp. 1501-1509). volume 1.

Duysinx, P., Van Miegroet, L., Lemaire, E., Brüls, O., \& Bruyneel, M. (2008). Topology and generalized shape optimization: Why stress constraints are so important? International Journal for Simulation and Multidisciplinary Design Optimization, 2, 253-258.

Eschenauer, H. A., Kobelev, V. V., \& Schumacher, A. (1994). Bubble method for topology and shape optimization of structures. Structural and Multidisciplinary Optimization, 8, 42-51.

Feijoo, R., Novotny, A., Taroco, E., \& Padra, C. (2005). The topologicalshape sensitivity method in two-dimensional linear elasticity topology design. Applications of Computational Mechanics in Structures and Fluids, .

Gao, J., Luo, Z., Xiao, M., Gao, L., \& Li, P. (2020a). A nurbs-based multimaterial interpolation (n-mmi) for isogeometric topology optimization of structures. Applied Mathematical Modelling, 81, 818-843.

Gao, J., Xiao, M., Gao, L., Yan, J., \& Yan, W. (2020b). Isogeometric topology optimization for computational design of re-entrant and chiral auxetic composites. Computer Methods in Applied Mechanics and Engineering, 362, 112876. 
Gao, X., Li, Y., Ma, H., \& Chen, G. (2020). Improving the overall performance of continuum structures: A topology optimization model considering stiffness, strength and stability. Computer Methods in Applied Mechanics and Engineering, 359, 112660.

Gao, X., \& Ma, H. (2015). Topology optimization of continuum structures under buckling constraints. Computers \& Structures, 157, 142-152.

Golub, G. H., \& Ye, Q. (2000). Inexact inverse iteration for generalized eigenvalue problems. BIT Numerical Mathematics, 40, 671-684.

Huang, X. \& Xie, M. (2010). Evolutionary topology optimization of continuum structures: methods and applications. John Wiley \& Sons.

Huang, X. \& Xie, Y. M. (2009). Bi-directional evolutionary topology optimization of continuum structures with one or multiple materials. Computational $\underline{\text { Mechanics, }}$ 43(3), 393.

Hughes, T. J., Levit, I., \& Winget, J. (1983). An element-by-element solution algorithm for problems of structural and solid mechanics. Computer Methods in Applied Mechanics and Engineering, 36, 241-254.

Ipsen, I. C. (1997). Computing an eigenvector with inverse iteration. SIAM review, 39, 254-291.

Kingman, J. J., Tsavdaridis, K., \& Toropov, V. (2015). Applications of topology optimisation in structural engineering: high-rise buildings \& steel components. Jordan Journal of Civil Engineering, 9, 335-357.

Kreisselmeier, G., \& Steinhauser, R. (1980). Systematic control design by optimizing a vector performance index. In Computer aided design of control systems (pp. 113-117). Elsevier.

Krishnakumar, A., \& Suresh, K. (2015). Hinge-free compliant mechanism design via the topological level-set. Journal of Mechanical Design, 137, 031406. 
Lehoucq, R. B., Sorensen, D. C., \& Yang, C. (1998). ARPACK users' guide: solution of large-scale eigenvalue problems with implicitly restarted Arnoldi methods. SIAM.

Lindgaard, E., \& Dahl, J. (2013). On compliance and buckling objective functions in topology optimization of snap-through problems. Structural and Multidisciplinary Optimization, 47, 409-421.

Luo, Q., \& Tong, L. (2015). Structural topology optimization for maximum linear buckling loads by using a moving iso-surface threshold method. Structural and Multidisciplinary Optimization, 52, 71-90.

Mirzendehdel, A. M. \& Suresh, K. (2015). A pareto-optimal approach to multimaterial topology optimization. Journal of Mechanical Design, 137(10).

Neves, M., Rodrigues, H., \& Guedes, J. (1995). Generalized topology design of structures with a buckling load criterion. Structural and Multidisciplinary Optimization, 10, 71-78.

Novotny, A., Feijóo, R., Taroco, E., \& Padra, C. (2007). Topological sensitivity analysis for three-dimensional linear elasticity problem. Computer Methods in Applied Mechanics and Engineering, 196, 4354-4364.

Ovtchinnikov, E. E. (2008). Computing several eigenpairs of hermitian problems by conjugate gradient iterations. Journal of Computational Physics, 227, 9477-9497.

Radel, S., Diourte, A., Soulié, F., Company, O., \& Bordreuil, C. (2019). Skeleton arc additive manufacturing with closed loop control. Additive Manufacturing, 26, 106-116.

Remouchamps, A., Bruyneel, M., Fleury, C., \& Grihon, S. (2011). Application of 660 a bi-level scheme including topology optimization to the design of an aircraft pylon. Structural and Multidisciplinary Optimization, 44, 739-750. 
Rodriguez, T., Montemurro, M., Le Texier, P., \& Pailhès, J. (2020). Structural displacement requirement in a topology optimization algorithm based on isogeometric entities. Journal of Optimization Theory and Applications, 184(1), $250-276$.

Saad, Y., Yeung, M., Erhel, J., \& Guyomarc'h, F. (2000). A deflated version of the conjugate gradient algorithm. SIAM Journal on Scientific Computing, 21, 1909-1926.

Sigmund, O. \& Petersson, J. (1998). Numerical instabilities in topology optimization: a survey on procedures dealing with checkerboards, meshdependencies and local minima. Structural optimization, 16(1), 68-75.

Shanglong, Z., Arun, L., NoratoDorn, J. A., \& S, W. (2020). Adaptive mesh refinement for topology optimization with discrete geometric components. Computer Methods in Applied Mechanics and Engineering, 364.

Sokolowski, J., \& Zochowski, A. (1999). On the topological derivative in shape optimization. SIAM journal on control and optimization, 37, 1251-1272.

Stolarski, T., Nakasone, Y., \& Yoshimoto, S. (2018). Engineering analysis with ANSYS software. Butterworth-Heinemann.

Suresh, K. (2010). A 199-line matlab code for pareto-optimal tracing in topology optimization. Structural and Multidisciplinary Optimization, 42, 665-679.

Suresh, K. (2013). Efficient generation of large-scale pareto-optimal topologies. Structural and Multidisciplinary Optimization, 47, 49-61.

Suresh, K., Ramani, A., \& Kaushik, A. (2012). An adaptive weighting strategy for multi-load topology optimization. In ASME 2012 International Design Engineering Technical Conferences \& Computers and Information in Engineering Conference.

Suresh, K., \& Takalloozadeh, M. (2013). Stress-constrained topology optimization: a topological level-set approach. Structural and Multidisciplinary Optimization, 48, 295-309. 
${ }_{690}$ Timoshenko, S., \& Gere, J. (1960). Theory of elastic stability. McGraw Hill Book Company.

Trefethen, L. N., \& Bau III, D. (1997). Numerical linear algebra volume 50. Siam.

Verbart, A., Langelaar, M., \& Van Keulen, F. (2017). A unified aggregation and relaxation approach for stress-constrained topology optimization. Structural and Multidisciplinary Optimization, 55, 663-679.

Wang, M. Y., Wang, X., \& Guo, D. (2003). A level set method for structural topology optimization. Computer methods in applied mechanics and engineering, 192(1), 227-246.

Yadav, P. \& Suresh, K. (2014). Large scale finite element analysis via assemblyfree deflated conjugate gradient. Journal of Computing and Information Science in Engineering, 14(4), 041008.

Zhu, J., Zhang, W., \& Xia, L. (2015). Topology optimization in aircraft and aerospace structures design. Archives of Computational Methods in Engineering . . doi $10.1007 / \mathrm{s} 11831-015-9151-2$. 